\title{
Search for Invisible Decays of a Higgs Boson Produced in Association with a $Z$ Boson in ATLAS
}

\author{
G. Aad et al.* \\ (ATLAS Collaboration)
}

(Received 13 February 2014; revised manuscript received 11 April 2014; published 20 May 2014)

\begin{abstract}
A search for evidence of invisible-particle decay modes of a Higgs boson produced in association with a $Z$ boson at the Large Hadron Collider is presented. No deviation from the standard model expectation is observed in $4.5 \mathrm{fb}^{-1}\left(20.3 \mathrm{fb}^{-1}\right)$ of 7 (8) TeV $p p$ collision data collected by the ATLAS experiment. Assuming the standard model rate for $Z H$ production, an upper limit of $75 \%$, at the $95 \%$ confidence level is set on the branching ratio to invisible-particle decay modes of the Higgs boson at a mass of $125.5 \mathrm{GeV}$. The limit on the branching ratio is also interpreted in terms of an upper limit on the allowed dark matter-nucleon scattering cross section within a Higgs-portal dark matter scenario. Within the constraints of such a scenario, the results presented in this Letter provide the strongest available limits for low-mass dark matter candidates. Limits are also set on an additional neutral Higgs boson, in the mass range $110<m_{H}<400 \mathrm{GeV}$, produced in association with a $Z$ boson and decaying to invisible particles.
\end{abstract}

DOI: 10.1103/PhysRevLett.112.201802

PACS numbers: 14.80.Bn, 12.60.Fr, 14.80.Ec, 95.35.+d

Some extensions of the standard model (SM) allow a Higgs boson [1-3] to decay to a pair of stable or long-lived particles [4-18] that are not observed by the ATLAS detector. For instance the Higgs boson can decay into two particles with very small interaction cross sections with SM particles, such as dark matter (DM) candidates. Collider data can be used to directly constrain the branching ratio of the Higgs boson to invisible particles. Similarly, limits can be placed on the cross section times branching ratio of any additional Higgs bosons decaying predominantly to invisible particles. LEP results [19] put limits on an invisibly decaying Higgs boson, produced in association with a $Z$ boson, for Higgs masses below $120 \mathrm{GeV}$.

This Letter presents a search for invisible decays of a Higgs boson produced in association with a $Z$ boson. A Higgs boson in the mass range $110<m_{H}<400 \mathrm{GeV}$ is considered. The distribution of the missing transverse momentum $\left(E_{T}^{\text {miss }}\right)$ in events with an electron or a muon pair consistent with a $Z$ boson decay is used to constrain the $\mathrm{ZH}$ production cross section times the branching ratio of the Higgs boson decaying to invisible particles, over the full mass range. For the newly discovered Higgs boson, a constraint could be placed on the branching ratio to invisible particles. In this case the mass of the Higgs boson is taken to be $m_{H}=125.5 \mathrm{GeV}$, the best-fit value from the ATLAS experiment [20], and the $Z H$ production cross section is assumed to be that predicted for the SM Higgs boson. This assumption implies that the hypothesized

* Full author list given at the end of the article.

Published by the American Physical Society under the terms of the Creative Commons Attribution 3.0 License. Further distribution of this work must maintain attribution to the author(s) and the published articles title, journal citation, and DOI. unobserved particles that couple to the Higgs boson have sufficiently weak couplings to other SM particles to not affect the Higgs boson production cross sections. The total cross section for the associated production of a SM Higgs boson, with $m_{H}=125.5 \mathrm{GeV}$, and a $Z$ boson, calculated to next-to-next-to-leading order in QCD [21] and including next-to-leading-order (NLO) electroweak corrections $[22,23]$, is $331 \mathrm{fb}$ at $\sqrt{s}=7 \mathrm{TeV}$ and $410 \mathrm{fb}$ at $\sqrt{s}=$ $8 \mathrm{TeV}$ [24]. The SM branching ratio of the Higgs boson decaying to invisible particles is $1.2 \times 10^{-3}$, arising from the $H \rightarrow Z Z^{(*)} \rightarrow 4 \nu$ decay. The present search is not sensitive to the low branching ratio for this decay, but instead searches for enhancements in the decay fraction to invisible particles due to physics beyond the standard model (BSM).

The search uses $4.5 \mathrm{fb}^{-1}$ of data recorded with the ATLAS detector in 2011 at $\sqrt{s}=7 \mathrm{TeV}$ and $20.3 \mathrm{fb}^{-1}$ of data recorded in 2012 at $\sqrt{s}=8 \mathrm{TeV}$. The ATLAS detector has been described elsewhere [25]. Simulated signal and background event samples are produced with Monte Carlo (MC) event generators, passed through a full GEANT4 [26] simulation of the ATLAS detector [27] and reconstructed with the same software as the data.

The signal samples are generated with HERWIG++ [28] and its internal POWHEG method [29,30]. The SM ZZ and $W Z$ backgrounds are taken from simulation, since they have limited statistics in the control regions that would allow us to estimate these backgrounds with data. All the other background processes to this search are determined from data. In these cases, simulated samples are only used as cross-checks for the obtained background estimates. POWHEG [29-31] interfaced with PYTHIA8 [32] is used to model SM $Z Z$ and $W Z$ production [33]. The production of $W W$ is modeled using HERWIG [34] and SHERPA [35] 
for the 7 and $8 \mathrm{TeV}$ data, respectively. A separate sample simulated with gg2VV [36] interfaced with JIMMY [37] accounts for $W W / Z Z$ production through quark-box diagrams, which are not included in the above mentioned samples. The MC@NLO [38] generator interfaced with JIMMY is used to model $t \bar{t}, W t$, and $s$-channel single topquark production. ACERMC [39] interfaced with PYTHIA [40] models $t$-channel single top-quark production. Inclusive $Z / \gamma *$ production is simulated with ALPGEN [41] interfaced with JIMMY or PYTHIA for the 7 or $8 \mathrm{TeV}$ data, respectively. Inclusive $W$ production is simulated with ALPGEN interfaced with JIMMY. Contributions to this search from the $H \rightarrow W W^{(*)} \rightarrow \ell \nu \ell \nu$ and $H \rightarrow Z Z^{(*)} \rightarrow$ $\ell \ell \nu \nu$ decays of a $125.5 \mathrm{GeV}$ SM Higgs boson are studied using POWHEG $[29-31,42,43]$ interfaced with PYTHIA8 and found to be negligible.

Electron candidates are reconstructed from isolated energy deposits in the electromagnetic calorimeter with a shower shape consistent with electrons or photons, matched to inner detector tracks [44]. The electrons used to form a $Z$ boson candidate are required to have transverse momentum $p_{\mathrm{T}}>20 \mathrm{GeV}$ and pseudorapidity $|\eta|<2.47$ [45]. Electrons with $p_{\mathrm{T}}>7 \mathrm{GeV}$ that satisfy less stringent identification criteria on the calorimeter cluster shape, track quality, and track-cluster matching [44] are used to veto events with more than two charged leptons.

Muon candidates are reconstructed combining tracks independently found in the muon spectrometer and inner tracking detector [46]. Muons forming a $Z$ boson candidate are required to have $p_{\mathrm{T}}>20 \mathrm{GeV}$ and $|\eta|<2$.4. Muons with $p_{\mathrm{T}}>7 \mathrm{GeV}$ are used to veto events with more than two charged leptons.

Jets are reconstructed using the anti- $k_{t}$ algorithm [47] with a radius parameter $R=0.4$. They must have $p_{T}>$ $20 \mathrm{GeV}$ and $|\eta|<4.5$. To discriminate against jets from additional minimum bias interactions, selection criteria are applied to ensure that most of the jet momentum, for jets with $|\eta|<2.5$, is associated with tracks originating from the primary vertex, which is taken to be the vertex with the highest summed $p_{T}^{2}$ of associated tracks.

To ensure good separation between electrons, muons, and jets, electrons are removed if they are within $\Delta R \leq 0.2$ of an identified muon, and jets are removed if they are within $\Delta R \leq 0.2$ of an identified electron. Remaining electrons and muons are removed if they are within $\Delta R \leq 0.4$ of a remaining jet or if the scalar sum of track momenta, not associated with the lepton, in a cone of $\Delta R<0.2$ around the lepton direction is greater than $10 \%$ of the lepton $p_{\mathrm{T}}$.

The $E_{T}^{\text {miss }}$ is the magnitude of the negative vectorial sum of the transverse momenta from calibrated objects, such as identified electrons, muons, photons, hadronic decays of tau leptons, and jets [48]. Clusters of calorimeter cells not matched to any object are also included. The analysis also uses a track-based missing transverse momentum $\left(p_{T}^{\text {miss }}\right)$ computed from all inner detector tracks with
$p_{T}>500 \mathrm{MeV}$ and $|\eta|<2.5$, that satisfy stringent quality criteria [49] and are consistent with originating from the primary vertex. For the $p_{T}^{\text {miss }}$ calculation, tracks matched to electrons are discarded and replaced by the transverse energy $E_{T}$ of the matched cluster measured in the calorimeter to include any photon radiation in the calculation.

Event selection criteria are determined in an optimization procedure, using simulated samples, to maximize the signal significance of the search. Events are required to pass a single-lepton or lepton-pair trigger, with small variations in the applied $p_{T}$ threshold in different data-taking periods. Events must also have at least one reconstructed vertex with at least three associated tracks with $p_{T}>500 \mathrm{MeV}$. Data quality criteria are applied to reject events from noncollision backgrounds or events with degraded detector performance [48].

The invariant mass of the selected dilepton system, $m_{\ell \ell}$, is required to satisfy $76<m_{\ell \ell}<106 \mathrm{GeV}$ to be consistent with leptons originating from a $Z$ boson decay.

Figure 1 shows the $E_{T}^{\text {miss }}$ distribution in the $8 \mathrm{TeV}$ data sample after the dilepton mass requirement. In this figure the data are consistent with the expected background based on simulated samples for all but the multijet background. The uncertainty band of the expected background is widest in the region dominated by the steeply falling $Z$ boson background. To reject the majority of this background, $E_{T}^{\text {miss }}$ is required to be greater than $90 \mathrm{GeV}$. In events where a significant $E_{T}^{\text {miss }}$ arises from misreconstructed energy in the calorimeter, the vectors of $E_{T}^{\text {miss }}$ and $p_{T}^{\text {miss }}$ are likely to have different azimuthal angles. Thus the azimuthal difference of these two vectors, $\Delta \phi\left(E_{T}^{\text {miss }}, p_{T}^{\text {miss }}\right)$, is required to be less than 0.2 .

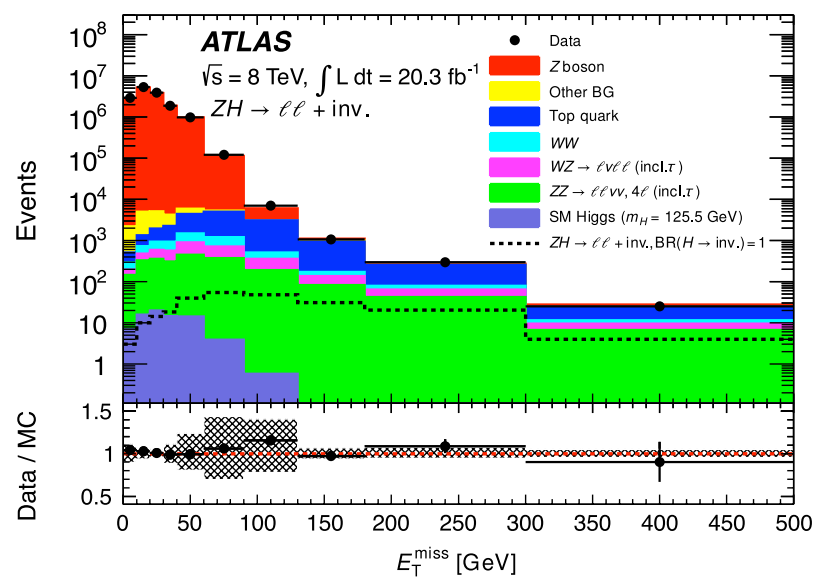

FIG. 1 (color online). Distribution of $E_{T}^{\text {miss }}$ for events with the invariant mass of the two leptons $76<m_{\ell \ell}<106 \mathrm{GeV}$ in the $8 \mathrm{TeV}$ data (dots). The stacked histograms represent the background predictions from simulation. The signal hypothesis is shown by a dotted line and assumes the SM $Z H$ production rate for a $m_{H}=125.5 \mathrm{GeV}$ Higgs boson with $\operatorname{BR}(H \rightarrow$ inv. $)=1$. The inset at the bottom of the figure shows the ratio of the data to the combined background expectations as well as a band corresponding to the combined systematic uncertainties. 
For the signal, the momentum of the reconstructed $Z$ boson is expected to be balanced by the momentum of the invisibly decaying Higgs boson. Therefore the azimuthal separation between the dilepton system, where the magnitude of its transverse momentum is defined as $p_{T}^{\ell \ell}$, and the $E_{T}^{\text {miss }}, \Delta \phi\left(p_{T}^{\ell \ell}, E_{T}^{\text {miss }}\right)$, is required to be greater than 2.6. The boost of the $Z$ boson causes the decay leptons to be produced with a small opening angle. The azimuthal opening angle of the two leptons, $\Delta \phi(\ell, \ell)$, is thus required to be less than 1.7. Furthermore $p_{T}^{\ell \ell}$ and $E_{T}^{\text {miss }}$ are expected to be similar. Therefore the fractional $p_{T}$ difference, defined as $\left|E_{T}^{\text {miss }}-p_{T}^{\ell \ell}\right| / p_{T}^{\ell \ell}$, is required to be less than 0.2 . Finally, for the majority of the signal no additional high- $p_{T}$ jets are expected to be observed in the events, while for the background from boosted $Z$ bosons and from $t \bar{t}$ pairs one or more jets are expected. Thus, events are required to have no reconstructed jets with $p_{T}>25 \mathrm{GeV}$ and $|\eta|<2.5$.

After the selection requirements, the dominant background is $\mathrm{SM} Z Z$ production followed by SM $W Z$ production, as shown in Table I. These backgrounds are simulated using MC samples normalized to NLO cross sections. The simulation of $W Z$ events is validated by comparing them to data events in which the third-lepton veto is replaced by an explicit third-lepton requirement. The theoretical prediction of the $Z Z$ production is in agreement with the ATLAS cross-section measurement at $\sqrt{s}=7 \mathrm{TeV}$ [50].

Background contributions from events with a genuine isolated lepton pair, not originating from a $Z \rightarrow e e$ or $Z \rightarrow$ $\mu \mu$ decay $(W W, t \bar{t}, W t$, and $Z \rightarrow \tau \tau)$, are estimated by exploiting the flavor symmetry in the dilepton final state of these processes. Distributions for events with an $e \mu$ pair, appropriately scaled to account for differences in electron and muon reconstruction efficiencies, can be used to estimate this background in the electron and muon channels. The difference between the efficiencies for electrons and muons is estimated using the square root of the ratio of the numbers of dimuon and dielectron events in data within the $m_{\ell \ell}$ window. Events in the $e \mu$ control region not originating from $W W, t \bar{t}, W t$, or $Z \rightarrow \tau \tau$ backgrounds are subtracted using simulated samples. Important sources of systematic uncertainty are variations in the correction factor for the efficiencies for electrons and muons and uncertainties in the simulated samples used for the subtraction. The combined systematic uncertainty is $23 \%$ for both the 7 and $8 \mathrm{TeV}$ data. The estimated background from these sources is consistent with the expectation from the simulation.

The background from inclusive $Z \rightarrow e e$ and $Z \rightarrow \mu \mu$ production in the signal region is estimated from the background in three sideband regions [51]. These sideband regions are formed by considering events failing one or both of the nominal selection requirements applied to $\Delta \phi\left(E_{T}^{\text {miss }}, p_{T}^{\text {miss }}\right)$ and the fractional $p_{T}$ difference. Contributions from non- $Z$ backgrounds in the sideband regions are subtracted. The impact from a correlation between the above two variables is determined from the simulation and a correction, of at most $7 \%$, is applied to account for it. The main uncertainties are due to variations in this correction and differences in the shape of the $E_{T}^{\text {miss }}$ distribution in the control regions. The overall systematic uncertainty is $52 \%$ in the $7 \mathrm{TeV}$ data and $59 \%$ in the $8 \mathrm{TeV}$ data.

The small background from events with only one genuine isolated lepton (inclusive $W$, single-lepton top pairs and single top production) or from multijet events is estimated from data using control samples, selected by requiring two lepton candidates of which at least one fails the full lepton selection criteria. These samples are scaled with a measured $p_{T}$-dependent factor, determined from data as described in Ref. [52]. Systematic uncertainties are determined following the procedures used in Ref. [52], yielding an uncertainty of $40 \%$ in the $7 \mathrm{TeV}$ data and $21 \%$ in the $8 \mathrm{TeV}$ data.

Systematic uncertainties on the signal and the SM ZZ and $W Z$ backgrounds are derived from the luminosity uncertainty, the propagation of reconstructed object uncertainties, and from theoretical uncertainties on the production cross sections. The luminosity uncertainty is $1.8 \%$ for the $7 \mathrm{TeV}$ data-taking period and $2.8 \%$ for the $8 \mathrm{TeV}$ datataking period [53].

Lepton trigger and identification efficiencies as well as the energy scale and resolution are determined from data using large samples of $Z$ events. After appropriate corrections to the simulation, uncertainties are propagated to the

TABLE I. Number of events observed in data and expected from the signal and from each background source for the 7 and $8 \mathrm{TeV}$ data-taking periods. Uncertainties on the signal and background expectations are presented with statistical uncertainties first and systematic uncertainties second.

\begin{tabular}{lcc}
\hline \hline Data period & $2011(7 \mathrm{TeV})$ & $2012(8 \mathrm{TeV})$ \\
\hline$Z Z \rightarrow \ell \ell \nu \nu$ & $20.0 \pm 0.7 \pm 1.6$ & $91 \pm 1 \pm 7$ \\
$W Z \rightarrow \ell \nu \ell \ell$ & $4.8 \pm 0.3 \pm 0.5$ & $26 \pm 1 \pm 3$ \\
Dileptonic $t \bar{t}, W t, W W, Z \rightarrow \tau \tau$ & $0.5 \pm 0.4 \pm 0.1$ & $20 \pm 3 \pm 5$ \\
$Z \rightarrow e e, Z \rightarrow \mu \mu$ & $0.13 \pm 0.12 \pm 0.07$ & $0.9 \pm 0.3 \pm 0.5$ \\
$W+$ jets, multijet, semileptonic top & $0.020 \pm 0.005 \pm 0.008$ & $0.29 \pm 0.02 \pm 0.06$ \\
Total background & $25.4 \pm 0.8 \pm 1.7$ & $138 \pm 4 \pm 9$ \\
Signal $\left(m_{H}=125.5 \mathrm{GeV}, \sigma_{Z H, S M}, \mathrm{BR}(H \rightarrow\right.$ inv. $\left.)=1\right)$ & $8.9 \pm 0.1 \pm 0.5$ & $44 \pm 1 \pm 3$ \\
Observed & 28 & 152 \\
\hline \hline
\end{tabular}


event selection. These uncertainties contribute typically $1.0 \%-1.5 \%$ to the overall selection uncertainty. Jet energy scale and resolution uncertainties are derived using a combination of techniques that use dijet, photon + jet, and $Z+$ jet events $[54,55]$. These contribute an uncertainty of between $3 \%$ and $6 \%$ on the final event selection. The uncertainties on the energy scale and resolution of leptons and jets are also propagated to the $E_{T}^{\text {miss }}$ calculation, and the resulting uncertainty in the latter is included in uncertainties given above. Uncertainties in the pile-up simulation, affecting in particular $E_{T}^{\text {miss }}$, contribute a further $1 \%-2 \%$ uncertainty.

Theoretical uncertainties on the $\mathrm{ZH}$ production cross section are derived from variations of the renormalization and factorization scale, $\alpha_{s}$, and the parton distribution functions (PDFs) [24]. These are combined to give an uncertainty of 3.6\%-5.7\% on the cross section. This analysis is sensitive to the distribution of the Higgs boson $p_{T}$ through the $E_{T}^{\text {miss }}$, and uncertainties in the $p_{T}$ boost of the Higgs boson can affect the signal yield. An additional systematic uncertainty of $1.9 \%$ is applied to the normalization $[22,23,56]$, and uncertainties as a function of the Higgs boson $p_{T}$ are considered as a systematic shape uncertainty.

The cross-section uncertainty on the ZZ background is 5\% from varying the PDFs, $\alpha_{s}$, and QCD scale. The uncertainty on the jet veto for the $Z Z$ background due to the parton showering is estimated to be $6.4 \%$ (5.5\%) for the 7 (8) $\mathrm{TeV}$ data. Because the $E_{T}^{\text {miss }}$ distribution of the final selected sample is used in the limit-setting procedure, the impact of PDFs, $\alpha_{s}$, and QCD scale uncertainties on the shape of this distribution is also considered. The theoretical uncertainty of the $W Z$ background is considered similarly. The total systematic uncertainty on the SM ZZ background is $8 \%$ for both the 7 and $8 \mathrm{TeV}$ data-taking periods, whereas for the $W Z$ background it is $10 \%$ (13\%) for the 7 (8) TeV data-taking periods.

Event reconstruction and theoretical uncertainties are considered as correlated between the 7 and $8 \mathrm{TeV}$ data, and between the signals and backgrounds estimated from simulation. The systematic uncertainties in methods that determine backgrounds from data using control regions are also assumed to be correlated between the two data sets. The luminosity uncertainty is considered as uncorrelated between the 7 and $8 \mathrm{TeV}$ data.

The numbers of observed and expected events for the 7 and $8 \mathrm{TeV}$ data-taking periods are shown in Table I. Figure 2 shows the $E_{T}^{\text {miss }}$ distribution after the full event selection for the $8 \mathrm{TeV}$ data and the expected backgrounds. The normalization of the backgrounds is extracted from a binned profile maximum likelihood fit in the signal region. Systematic uncertainties are considered as nuisance parameters, and are assumed to be constrained by Gaussian distributions. The signal expectation shown corresponds to a Higgs boson with $m_{H}=125.5 \mathrm{GeV}$, a SM $Z H$ production rate, and $\operatorname{BR}(H \rightarrow$ inv. $)=1$. No significant excess is observed over the SM expectation.

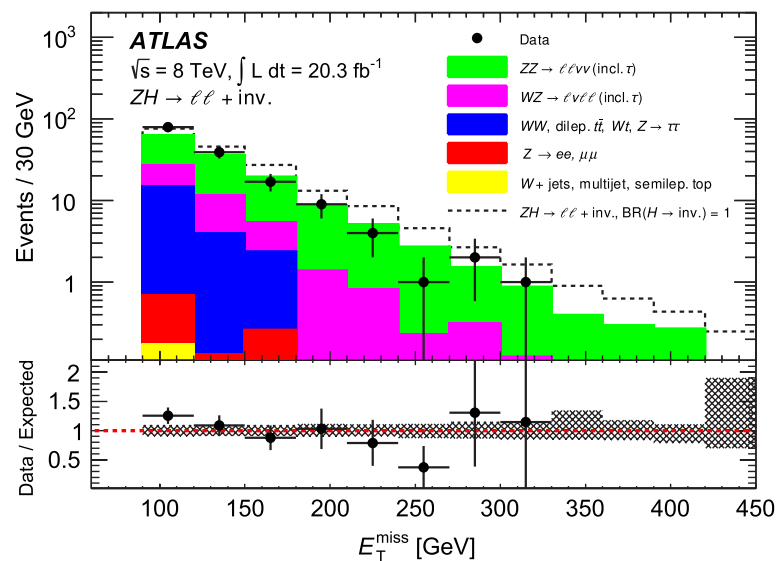

FIG. 2 (color online). Distribution of $E_{T}^{\text {miss }}$ after the full selection in the $8 \mathrm{TeV}$ data (dots). The filled stacked histograms represent the background expectations. The signal expectation for a Higgs boson with $m_{H}=125.5 \mathrm{GeV}$, a SM $Z H$ production rate and $\operatorname{BR}(H \rightarrow$ inv. $)=1$ is stacked on top of the background expectations. The inset at the bottom of the figure shows the ratio of the data to the combined background expectations. The hashed area shows the systematic uncertainty on the combined background expectation.

Limits are set on the cross section times branching ratio for a Higgs boson decaying to invisible particles anywhere in the mass range $110<m_{H}<400 \mathrm{GeV}$. The limits are computed using a maximum likelihood fit to the $E_{T}^{\text {miss }}$ distribution following the $\mathrm{CL}_{s}$ (signal confidence level) modified frequentist formalism [57] with a profile likelihood test statistic [58]. Figure 3 shows the 95\% C.L. upper limits on $\sigma_{Z H} \times \mathrm{BR}(H \rightarrow$ inv. $)$ in the mass range $110<m_{H}<400 \mathrm{GeV}$ for the combined 7 and $8 \mathrm{TeV}$ data. The expectation for a Higgs boson with a production cross section equal to that expected for a SM Higgs boson and $\operatorname{BR}(H \rightarrow$ inv. $)=1$ is also shown.

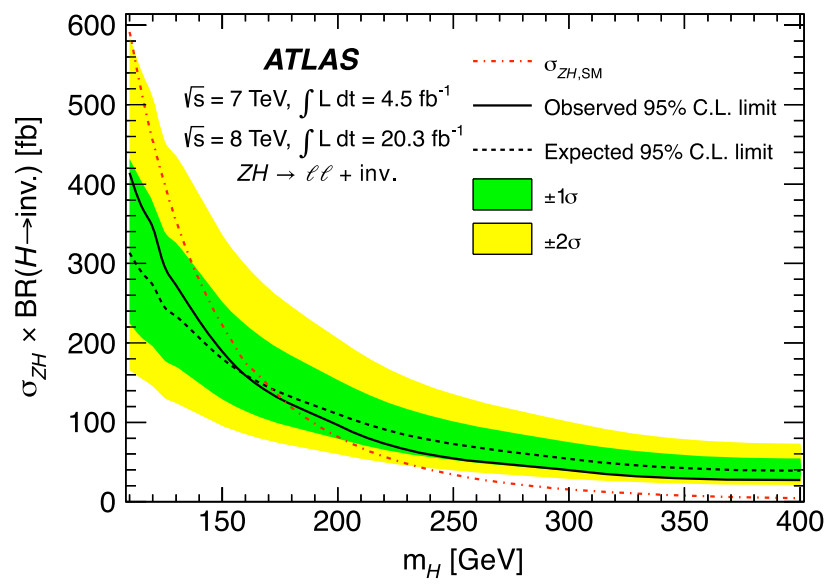

FIG. 3 (color online). Upper limits on $\sigma_{Z H} \times \mathrm{BR}(H \rightarrow$ inv.) at $95 \%$ C.L. for a Higgs boson with $110<m_{H}<400 \mathrm{GeV}$, for the combined 7 and $8 \mathrm{TeV}$ data. The full and dashed lines show the observed and expected limits, respectively. 
For the discovered Higgs boson an upper limit of $75 \%$ at 95\% C.L. (63\% at $90 \%$ C.L.) is set on the branching ratio to invisible particles. For this the predicted SM $Z H$ production rate with $m_{H}=125.5 \mathrm{GeV}$, is assumed. The expected limit in the absence of BSM decays to invisible particles is $62 \%$ at $95 \%$ C.L. (52\% at $90 \%$ C.L.).

Within the context of a Higgs-portal DM scenario [59], in which the Higgs boson acts as the mediator particle between DM and SM particles, the Higgs boson can decay to a pair of DM particles. In this case the limit on $\mathrm{BR}(H \rightarrow$ inv. $)$ for the $125.5 \mathrm{GeV}$ Higgs boson can be interpreted in terms of an upper limit on the DM-nucleon scattering cross section [60]. The formalism used to interpret the $\operatorname{BR}(H \rightarrow$ inv. $)$ limit in terms of the spin-independent DM-nucleon scattering cross sections is described in Refs. [61,62]. Figure 4 shows $90 \%$ C.L. upper limits on the DM-nucleon scattering cross section for three model variants in which a single DM candidate is considered and is either a scalar, a vector, or a Majorana fermion. The Higgs-nucleon coupling is taken as $0.33_{-0.07}^{+0.30}[62]$, the uncertainty of which is expressed by the bands in the figure. Spin-independent results from directsearch experiments are also shown [63-70]. These results do not depend on the assumptions of the Higgs-portal scenario. Within the constraints of such a scenario, however, the results presented in this Letter provide the strongest available limits for low-mass DM candidates. There is no sensitivity to these models once the mass of the DM candidate exceeds $m_{H} / 2$. A search by the ATLAS experiment for DM in more generic models, also using the dilepton $+\operatorname{large} E_{T}^{\text {miss }}$ final state, is presented in Ref. [71].

We thank CERN for the very successful operation of the LHC, as well as the support staff from our institutions without whom ATLAS could not be operated efficiently.

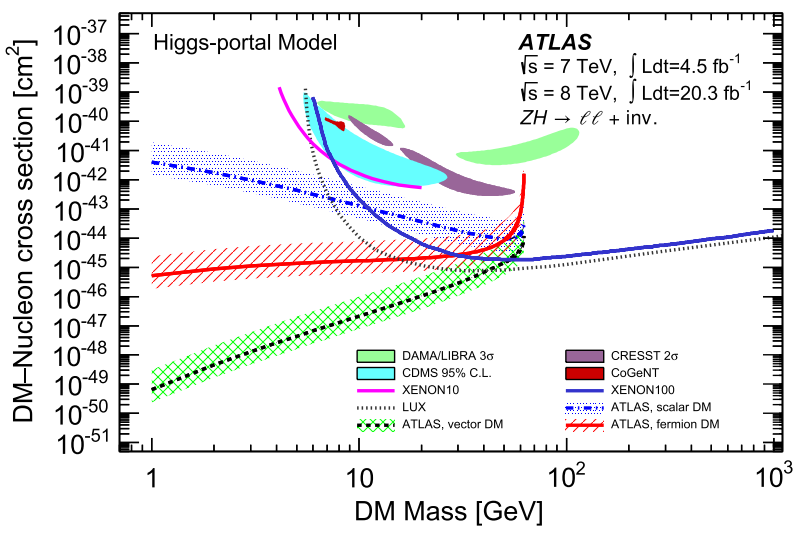

FIG. 4 (color online). Limits on the DM-nucleon scattering cross section at $90 \%$ C.L., extracted from the $\mathrm{BR}(H \rightarrow$ inv. $)$ limit in a Higgs-portal scenario, compared to results from direct-search experiments [63-70]. Cross-section limits and favored regions correspond to a $90 \%$ C.L., unless stated otherwise in the legend. Favored regions for DAMA and CoGeNT are based on Ref. [68]. The results from the direct-search experiments do not depend on the assumptions of the Higgs-portal scenario.
We acknowledge the support of ANPCyT, Argentina; YerPhI, Armenia; ARC, Australia; BMWF and FWF, Austria; ANAS, Azerbaijan; SSTC, Belarus; CNPq and FAPESP, Brazil; NSERC, NRC and CFI, Canada; CERN; CONICYT, Chile; CAS, MOST and NSFC, China; COLCIENCIAS, Colombia; MSMT CR, MPO CR and VSC CR, Czech Republic; DNRF, DNSRC and Lundbeck Foundation, Denmark; EPLANET, ERC and NSRF, European Union; IN2P3-CNRS, CEA-DSM/IRFU, France; GNSF, Georgia; BMBF, DFG, HGF, MPG and AvH Foundation, Germany; GSRT and NSRF, Greece; ISF, MINERVA, GIF, DIP and Benoziyo Center, Israel; INFN, Italy; MEXT and JSPS, Japan; CNRST, Morocco; FOM and NWO, Netherlands; BRF and RCN, Norway; MNiSW and NCN, Poland; GRICES and FCT, Portugal; MNE/IFA, Romania; MES of Russia and ROSATOM, Russian Federation; JINR; MSTD, Serbia; MSSR, Slovakia; ARRS and MIZŠ, Slovenia; DST/NRF, South Africa; MINECO, Spain; SRC and Wallenberg Foundation, Sweden; SER, SNSF and Cantons of Bern and Geneva, Switzerland; NSC, Taiwan; TAEK, Turkey; STFC, the Royal Society and Leverhulme Trust, United Kingdom; DOE and NSF, United States of America. The crucial computing support from all WLCG partners is acknowledged gratefully, in particular from CERN and the ATLAS Tier-1 facilities at TRIUMF (Canada), NDGF (Denmark, Norway, Sweden), CC-IN2P3 (France), KIT/GridKA (Germany), INFN-CNAF (Italy), NL-T1 (Netherlands), PIC (Spain), ASGC (Taiwan), RAL (UK) and BNL (USA) and in the Tier-2 facilities worldwide.

[1] F. Englert and R. Brout, Phys. Rev. Lett. 13, 321 (1964).

[2] P. W. Higgs, Phys. Rev. Lett. 13, 508 (1964).

[3] G. Guralnik, C. Hagen, and T. Kibble, Phys. Rev. Lett. 13, 585 (1964).

[4] K. Griest and H. E. Haber, Phys. Rev. D 37, 719 (1988).

[5] A. Djouadi, P. Janot, J. Kalinowski, and P. M. Zerwas, Phys. Lett. B 376, 220 (1996).

[6] I. Antoniadis, M. Tuckmantel, and F. Zwirner, Nucl. Phys. B707, 215 (2005).

[7] T. Binoth and J. J. van der Bij, Z. Phys. C 75, 17 (1997).

[8] Y. Chikashige, R. N. Mohapatra, and R. D. Peccei, Phys. Lett. 98B, 265 (1981).

[9] A. S. Joshipura and S. D. Rindani, Phys. Rev. Lett. 69, 3269 (1992).

[10] R. E. Shrock and M. Suzuki, Phys. Lett. 110B, 250 (1982).

[11] R. N. Mohapatra and J. W. F. Valle, Phys. Rev. D 34, 1642 (1986).

[12] M. C. Gonzalez-Garcia and J. W. F. Valle, Phys. Lett. B 216, 360 (1989).

[13] E. D. Carlson and L. J. Hall, Phys. Rev. D 40, 3187 (1989).

[14] L.-F. Li, Y. Liu, and L. Wolfenstein, Phys. Lett. 159B, 45 (1985).

[15] A. Zee, Phys. Lett. 93B, 389 (1980).

[16] F. de Campos, O. J. P. Éboli, J. Rosiek, and J. W. F. Valle, Phys. Rev. D 55, 1316 (1997). 
[17] S. P. Martin and J. D. Wells, Phys. Rev. D 60, 035006 (1999).

[18] G. F. Giudice, R. Rattazzi, and J. D. Wells, Nucl. Phys. B595, 250 (2001).

[19] LEP Higgs Working Group, Report No. LHWG Note 200106, 2001.

[20] ATLAS Collaboration, Phys. Lett. B 726, 88 (2013).

[21] O. Brein, A. Djouadi, and R. Harlander, Phys. Lett. B 579, 149 (2004).

[22] M. L. Ciccolini, S. Dittmaier, and M. Krämer, Phys. Rev. D 68, 073003 (2003).

[23] A. Denner, S. Dittmaier, S. Kallweit, and A. Mück, J. High Energy Phys. 03 (2012) 075.

[24] LHC Higgs Cross Section Working Group, CERN Report No. CERN-2013-004, edited by S. Dittmaier, C. Mariotti, G. Passarino, and R. Tanaka, 2013.

[25] ATLAS Collaboration, JINST 3, S08003 (2008).

[26] S. Agostinelli et al. (GEANT4 Collaboration), Nucl. Instrum. Methods Phys. Res., Sect. A 506, 250 (2003).

[27] ATLAS Collaboration, Eur. Phys. J. C 70, 823 (2010).

[28] M. Bähr et al., Eur. Phys. J. C 58, 639 (2008).

[29] P. Nason, J. High Energy Phys. 11 (2004) 040.

[30] S. Frixione, P. Nason, and C. Oleari, J. High Energy Phys. 11 (2007) 070.

[31] S. Alioli, P. Nason, C. Oleari, and E. Re, J. High Energy Phys. 06 (2010) 043.

[32] T. Sjöstrand, S. Mrenna, and P. Skands, Comput. Phys. Commun. 178, 852 (2008).

[33] T. Melia, P. Nason, R. Röntsch, and G. Zanderighi, J. High Energy Phys. 11 (2011) 078.

[34] G. Corcella, I. G. Knowles, G. Marchesini, S. Moretti, K. Odagiri, P. Richardson, M. H. Seymour, and B. R. Webber, J. High Energy Phys. 01 (2001) 010.

[35] T. Gleisberg, S. Höche, F. Krauss, M. Schönherr, S. Schumann, F. Siegert, and J. Winter, J. High Energy Phys. 02 (2009) 007.

[36] T. Binoth, M. Ciccolini, N. Kauer, and M. Krämer, J. High Energy Phys. 12 (2006) 046.

[37] J. M. Butterworth, J. R. Forshaw, and M. H. Seymour, Z. Phys. C 72, 637 (1996).

[38] S. Frixione and B. R. Webber, J. High Energy Phys. 06 (2002) 029.

[39] B. P. Kersevan and E. Richter-Was, Comput. Phys. Commun. 184, 919 (2013).

[40] T. Sjöstrand, S. Mrenna and P. Skands, J. High Energy Phys. 05 (2006) 026.

[41] M. L. Mangano, F. Piccinini, A. D. Polosa, M. Moretti, and R. Pittau, J. High Energy Phys. 07 (2003) 001.

[42] S. Alioli, P. Nason, C. Oleari, and E. Re, J. High Energy Phys. 04 (2009) 002.
[43] P. Nason and C. Oleari, J. High Energy Phys. 02 (2010) 037.

[44] ATLAS Collaboration, Eur. Phys. J. C 72, 1909 (2012).

[45] ATLAS uses a right-handed coordinate system with its origin at the nominal interaction point in the center of the detector and the $z$ axis along the beam pipe. Polar coordinates $(r, \phi)$ are used in the transverse plane, $\phi$ being the azimuthal angle around the beam pipe. The pseudorapidity $\eta$ is defined in terms of the polar angle $\theta$ as $\eta=-\ln \tan (\theta / 2)$, and $\Delta R=\sqrt{(\Delta \eta)^{2}+(\Delta \phi)^{2}}$.

[46] ATLAS Collaboration, J. High Energy Phys. 12 (2010) 060.

[47] M. Cacciari, G. P. Salam, and G. Soyez, J. High Energy Phys. 04 (2008) 063.

[48] ATLAS Collaboration, Eur. Phys. J. C 72, 1844 (2012).

[49] ATLAS Collaboration, Report No. ATLAS-CONF-2010020, 2010.

[50] ATLAS Collaboration, J. High Energy Phys. 03 (2013) 128.

[51] ATLAS Collaboration, Eur. Phys. J. C 71, 1577 (2011).

[52] ATLAS Collaboration, Phys. Rev. Lett. 107, 041802 (2011).

[53] ATLAS Collaboration, Eur. Phys. J. C 73, 2518 (2013).

[54] ATLAS Collaboration, Eur. Phys. J. C 73, 2304 (2013).

[55] ATLAS Collaboration, Eur. Phys. J. C 73, 2306 (2013).

[56] ATLAS Collaboration, Phys. Lett. B 718, 369 (2012).

[57] A. L. Read, J. Phys. G 28, 2693 (2002).

[58] G. Cowan, K. Cranmer, E. Gross, and O. Vitells, Eur. Phys. J. C 71, 1554 (2011).

[59] B. Patt and F. Wilczek, arXiv:hep-ph/0605188.

[60] P. J. Fox, R. Harnik, J. Kopp, and Y. Tsai, Phys. Rev. D 85, 056011 (2012).

[61] S. Kanemura, S. Matsumoto, T. Nabeshima, and N. Okada, Phys. Rev. D 82, 055026 (2010).

[62] A. Djouadi, O. Lebedev, Y. Mambrini, and J. Quevillon, Phys. Lett. B 709, 65 (2012).

[63] R. Bernabei et al., Eur. Phys. J. C 56, 333 (2008).

[64] J. Angle et al. (XENON10 Collaboration), Phys. Rev. Lett. 107, 051301 (2011).

[65] E. Aprile et al. (XENON100 Collaboration), Phys. Rev. Lett. 109, 181301 (2012).

[66] G. Angloher et al., Eur. Phys. J. C 72, 1971 (2012).

[67] C. E. Aalseth et al. (CoGeNT Collaboration), Phys. Rev. Lett. 107, 141301 (2011).

[68] P. J. Fox, J. Kopp, M. Lisanti, and N. Weiner, Phys. Rev. D 85, 036008 (2012).

[69] R. Agnese et al. (CDMS Collaboration), Phys. Rev. Lett. 111, 251301 (2013).

[70] D. S. Akerib et al. (LUX Collaboration), Phys. Rev. Lett. 112, 091303 (2014).

[71] ATLAS Collaboration, arXiv:1404.0051 [Phys. Rev. D (to be published)].

G. Aad, ${ }^{48}$ T. Abajyan, ${ }^{21}$ B. Abbott, ${ }^{112}$ J. Abdallah, ${ }^{152}$ S. Abdel Khalek, ${ }^{116}$ O. Abdinov, ${ }^{11}$ R. Aben, ${ }^{106}$ B. Abi, ${ }^{113}$ M. Abolins, ${ }^{89}$ O. S. AbouZeid, ${ }^{159}$ H. Abramowicz, ${ }^{154}$ H. Abreu, ${ }^{137}$ Y. Abulaiti, ${ }^{147 a, 147 b}$ B. S. Acharya, ${ }^{165 a, 165 b, b}$ L. Adamczyk ${ }^{38 a}$ D. L. Adams, ${ }^{25}$ T. N. Addy, ${ }^{56}$ J. Adelman, ${ }^{177}$ S. Adomeit, ${ }^{99}$ T. Adye, ${ }^{130}$ S. Aefsky, ${ }^{23}$ T. Agatonovic-Jovin, ${ }^{13 b}$ J. A. Aguilar-Saavedra, ${ }^{125 f, 125 a}$ M. Agustoni, ${ }^{17}$ S. P. Ahlen, ${ }^{22}$ A. Ahmad, ${ }^{149}$ F. Ahmadov, ${ }^{64, c}$ G. Aielli, ${ }^{134 a, 134 b}$ T. P. A. Åkesson, ${ }^{80}$ G. Akimoto, ${ }^{156}$ A. V. Akimov, ${ }^{95}$ M. A. Alam, ${ }^{76}$ J. Albert, ${ }^{170}$ S. Albrand, ${ }^{55}$ M. J. Alconada Verzini, ${ }^{70}$ M. Aleksa, ${ }^{30}$ I. N. Aleksandrov, ${ }^{64}$ F. Alessandria,${ }^{90 a}$ C. Alexa, ${ }^{26 a}$ G. Alexander, ${ }^{154}$ G. Alexandre, ${ }^{49}$ 
T. Alexopoulos, ${ }^{10}$ M. Alhroob, ${ }^{165 a, 165 c}$ G. Alimonti, ${ }^{90 a}$ L. Alio, ${ }^{84}$ J. Alison, ${ }^{31}$ B. M. M. Allbrooke, ${ }^{18}$ L. J. Allison, ${ }^{71}$ P. P. Allport, ${ }^{73}$ S. E. Allwood-Spiers, ${ }^{53}$ J. Almond, ${ }^{83}$ A. Aloisio, ${ }^{103 a, 103 b}$ R. Alon, ${ }^{173}$ A. Alonso, ${ }^{36}$ F. Alonso, ${ }^{70}$ A. Altheimer, ${ }^{35}$ B. Alvarez Gonzalez ${ }^{89}$ M. G. Alviggi, ${ }^{103 a, 103 b}$ K. Amako, ${ }^{65}$ Y. Amaral Coutinho, ${ }^{24 a}$ C. Amelung, ${ }^{23}$ V. V. Ammosov, ${ }^{129, a}$ S. P. Amor Dos Santos, ${ }^{125 a, 125 \mathrm{c}}$ A. Amorim, ${ }^{125 a, 125 \mathrm{~b}}$ S. Amoroso, ${ }^{48}$ N. Amram, ${ }^{154}$ G. Amundsen, ${ }^{23}$

C. Anastopoulos ${ }^{30}$ L. S. Ancu, ${ }^{17}$ N. Andari, ${ }^{30}$ T. Andeen, ${ }^{35}$ C. F. Anders, ${ }^{58 b}$ G. Anders, ${ }^{58 \mathrm{a}}$ K. J. Anderson, ${ }^{31}$

A. Andreazza, ${ }^{90 a, 90 b}$ V. Andrei, ${ }^{58 a}$ X. S. Anduaga ${ }^{70}$ S. Angelidakis, ${ }^{9}$ P. Anger, ${ }^{44}$ A. Angerami, ${ }^{35}$ F. Anghinolfi, ${ }^{30}$ A. V. Anisenkov, ${ }^{108}$ N. Anjos, ${ }^{125 a}$ A. Annovi, ${ }^{47}$ A. Antonaki, ${ }^{9}$ M. Antonelli, ${ }^{47}$ A. Antonov, ${ }^{97}$ J. Antos,${ }^{145 b}$ F. Anulli, ${ }^{133 a}$ M. Aoki, ${ }^{65}$ L. Aperio Bella, ${ }^{18}$ R. Apolle, ${ }^{119, \mathrm{~d}}$ G. Arabidze, ${ }^{89}$ I. Aracena, ${ }^{144}$ Y. Arai,${ }^{65}$ A. T. H. Arce, ${ }^{45}$ J-F. Arguin, ${ }^{94}$ S. Argyropoulos, ${ }^{42}$ E. Arik, ${ }^{19 a, a}$ M. Arik, ${ }^{19 a}$ A. J. Armbruster, ${ }^{88}$ O. Arnaez,${ }^{82}$ V. Arnal, ${ }^{81}$ O. Arslan, ${ }^{21}$ A. Artamonov, ${ }^{96}$ G. Artoni, ${ }^{23}$ S. Asai, ${ }^{156}$ N. Asbah, ${ }^{94}$ S. Ask, ${ }^{28}$ B. Åsman, ${ }^{147 a, 147 b}$ L. Asquith, ${ }^{6}$ K. Assamagan,,${ }^{25}$ R. Astalos, ${ }^{145 a}$ A. Astbury, ${ }^{170}$ M. Atkinson, ${ }^{166}$ N. B. Atlay, ${ }^{142}$ B. Auerbach, ${ }^{6}$ E. Auge, ${ }^{116}$ K. Augsten, ${ }^{127}$ M. Aurousseau, ${ }^{146 b}$ G. Avolio, ${ }^{30}$ G. Azuelos, ${ }^{94, e}$ Y. Azuma ${ }^{156}$ M. A. Baak,${ }^{30}$ C. Bacci, ${ }^{135 a, 135 b}$ A. M. Bach,${ }^{15}$ H. Bachacou,${ }^{137}$ K. Bachas,${ }^{155}$ M. Backes,${ }^{30}$ M. Backhaus,${ }^{21}$

J. Backus Mayes, ${ }^{144}$ E. Badescu, ${ }^{26 a}$ P. Bagiacchi, ${ }^{133 a, 133 b}$ P. Bagnaia, ${ }^{133 a, 133 b}$ Y. Bai, ${ }^{33 a}$ D. C. Bailey, ${ }^{159}$ T. Bain, ${ }^{35}$ J. T. Baines, ${ }^{130}$ O. K. Baker, ${ }^{177}$ S. Baker, ${ }^{77}$ P. Balek,,${ }^{128}$ F. Balli, ${ }^{137}$ E. Banas,,${ }^{39}$ Sw. Banerjee, ${ }^{174}$ D. Banfi, ${ }^{30}$ A. Bangert, ${ }^{151}$ V. Bansal,${ }^{170}$ H. S. Bansil, ${ }^{18}$ L. Barak, ${ }^{173}$ S. P. Baranov, ${ }^{95}$ T. Barber, ${ }^{48}$ E. L. Barberio, ${ }^{87}$ D. Barberis, ${ }^{50,50 b}$ M. Barbero, ${ }^{84}$ T. Barillari, ${ }^{100}$ M. Barisonzi, ${ }^{176}$ T. Barklow, ${ }^{144}$ N. Barlow, ${ }^{28}$ B. M. Barnett, ${ }^{130}$ R. M. Barnett, ${ }^{15}$ A. Baroncelli, ${ }^{135 a}$ G. Barone, ${ }^{49}$ A. J. Barr, ${ }^{119}$ F. Barreiro, ${ }^{81}$ J. Barreiro Guimarães da Costa ${ }^{57}$ R. Bartoldus, ${ }^{144}$ A. E. Barton, ${ }^{71}$ P. Bartos, ${ }^{145 a}$ V. Bartsch, ${ }^{150}$ A. Bassalat, ${ }^{16}$ A. Basye, ${ }^{166}$ R. L. Bates,${ }^{53}$ L. Batkova, ${ }^{145 a}$ J. R. Batley, ${ }^{28}$ M. Battistin, ${ }^{30}$ F. Bauer, ${ }^{137}$ H. S. Bawa, ${ }^{144, f}$ T. Beau, ${ }^{79}$ P. H. Beauchemin, ${ }^{162}$ R. Beccherle, ${ }^{123 a, 123 b}$ P. Bechtle, ${ }^{21}$ H. P. Beck, ${ }^{17}$ K. Becker, ${ }^{176}$ S. Becker, ${ }^{99}$ M. Beckingham, ${ }^{139}$ A. J. Beddall, ${ }^{19 c}$ A. Beddall, ${ }^{19 c}$ S. Bedikian, ${ }^{177}$ V. A. Bednyakov, ${ }^{64}$ C. P. Bee, ${ }^{149}$ L. J. Beemster, ${ }^{106}$ T. A. Beermann, ${ }^{176}$ M. Begel, ${ }^{25}$ K. Behr, ${ }^{119}$ C. Belanger-Champagne, ${ }^{86}$ P. J. Bell, ${ }^{49}$ W. H. Bell, ${ }^{49}$ G. Bella, ${ }^{154}$

L. Bellagamba, ${ }^{20 \mathrm{a}}$ A. Bellerive, ${ }^{29} \mathrm{M}$. Bellomo, ${ }^{85}$ A. Belloni, ${ }^{57}$ O. L. Beloborodova, ${ }^{108, g} \mathrm{~K}$. Belotskiy, ${ }^{97}$ O. Beltramello, ${ }^{30}$

O. Benary, ${ }^{154}$ D. Benchekroun, ${ }^{136 a}$ K. Bendtz, ${ }^{147 a, 147 b}$ N. Benekos, ${ }^{166}$ Y. Benhammou, ${ }^{154}$ E. Benhar Noccioli ${ }^{49}$

J. A. Benitez Garcia, ${ }^{160 b}$ D. P. Benjamin, ${ }^{45}$ J. R. Bensinger,${ }^{23}$ K. Benslama,${ }^{131}$ S. Bentvelsen, ${ }^{106}$ D. Berge, ${ }^{106}$

E. Bergeaas Kuutmann, ${ }^{16}$ N. Berger, ${ }^{5}$ F. Berghaus, ${ }^{170}$ E. Berglund, ${ }^{106}$ J. Beringer ${ }^{15}$ C. Bernard, ${ }^{22}$ P. Bernat, ${ }^{77}$ C. Bernius, ${ }^{78}$ F. U. Bernlochner, ${ }^{170}$ T. Berry, ${ }^{76}$ P. Berta, ${ }^{128}$ C. Bertella, ${ }^{84}$ F. Bertolucci, ${ }^{123 a, 123 b}$ M. I. Besana, ${ }^{90 a}$ G. J. Besjes, ${ }^{105}$ O. Bessidskaia, ${ }^{147 a, 147 b}$ N. Besson, ${ }^{137}$ S. Bethke, ${ }^{100}$ W. Bhimji ${ }^{46}$ R. M. Bianchi,${ }^{124}$ L. Bianchini, ${ }^{23}$ M. Bianco, ${ }^{30}$ O. Biebel,${ }^{99}$ S. P. Bieniek, ${ }^{77}$ K. Bierwagen,${ }^{54}$ J. Biesiada, ${ }^{15}$ M. Biglietti, ${ }^{135 a}$ J. Bilbao De Mendizabal, ${ }^{49}$ H. Bilokon, ${ }^{47}$ M. Bindi, ${ }^{20 a, 20 b}$ S. Binet, ${ }^{116}$ A. Bingul, ${ }^{19 \mathrm{c}}$ C. Bini, ${ }^{133 a, 133 \mathrm{~b}}$ B. Bittner, ${ }^{100}$ C. W. Black, ${ }^{151}$ J. E. Black, ${ }^{144}$ K. M. Black, ${ }^{22}$ D. Blackburn, ${ }^{139}$ R. E. Blair, ${ }^{6}$ J.-B. Blanchard, ${ }^{137}$ T. Blazek, ${ }^{145 a}$ I. Bloch, ${ }^{42}$ C. Blocker, ${ }^{23}$ W. Blum, ${ }^{82, a}$ U. Blumenschein,${ }^{54}$ G. J. Bobbink, ${ }^{106}$ V. S. Bobrovnikov, ${ }^{108}$ S. S. Bocchetta, ${ }^{80}$ A. Bocci, ${ }^{45}$ C. R. Boddy, ${ }^{119}$ M. Boehler, ${ }^{48}$ J. Boek, ${ }^{176}$ T. T. Boek, ${ }^{176}$ J. A. Bogaerts, ${ }^{30}$ A. G. Bogdanchikov, ${ }^{108}$ A. Bogouch, ${ }^{91, a}$ C. Bohm, ${ }^{147 a}$ J. Bohm, ${ }^{126}$ V. Boisvert, ${ }^{76}$ T. Bold ${ }^{38 a}$ V. Boldea, ${ }^{26 a}$ A. S. Boldyrev, ${ }^{98}$ N. M. Bolnet,${ }^{137}$ M. Bomben,${ }^{79}$ M. Bona,${ }^{75}$ M. Boonekamp,${ }^{137}$ C. Borer, ${ }^{17}$ A. Borisov,${ }^{129}$ G. Borissov,${ }^{71}$ M. Borri, ${ }^{83}$ S. Borroni, ${ }^{42}$ J. Bortfeldt ${ }^{99}$ V. Bortolotto, ${ }^{135 a, 135 b}$ K. Bos, ${ }^{106}$ D. Boscherini, ${ }^{20 a}$ M. Bosman, ${ }^{12}$ H. Boterenbrood, ${ }^{106}$

J. Bouchami, ${ }^{94}$ J. Boudreau, ${ }^{124}$ E. V. Bouhova-Thacker, ${ }^{71}$ D. Boumediene, ${ }^{34}$ C. Bourdarios, ${ }^{116}$ N. Bousson, ${ }^{84}$ S. Boutouil, ${ }^{136 \mathrm{~d}}$ A. Boveia, ${ }^{31}$ J. Boyd ${ }^{30}$ I. R. Boyko, ${ }^{64}$ I. Bozovic-Jelisavcic, ${ }^{13 \mathrm{~b}}$ J. Bracinik, ${ }^{18}$ P. Branchini, ${ }^{135 \mathrm{a}}$ A. Brandt, ${ }^{8}$ G. Brandt, ${ }^{15}$ O. Brandt, ${ }^{58 a}$ U. Bratzler, ${ }^{157}$ B. Brau, ${ }^{85}$ J. E. Brau, ${ }^{115}$ H. M. Braun, ${ }^{176, a}$ S. F. Brazzale, ${ }^{165 a, 165 c}$ B. Brelier, ${ }^{159}$ K. Brendlinger, ${ }^{121}$ A. J. Brennan, ${ }^{87}$ R. Brenner, ${ }^{167}$ S. Bressler ${ }^{173}$ T. M. Bristow, ${ }^{46}$ D. Britton, ${ }^{53}$ F. M. Brochu, ${ }^{28}$ I. Brock, ${ }^{21}$ R. Brock, ${ }^{89}$ F. Broggi, ${ }^{90 a}$ C. Bromberg, ${ }^{89}$ J. Bronner, ${ }^{100}$ G. Brooijmans, ${ }^{35}$ T. Brooks, ${ }^{76}$ W. K. Brooks, ${ }^{32 b}$ J. Brosamer, ${ }^{15}$ E. Brost, ${ }^{115}$ G. Brown, ${ }^{83}$ J. Brown, ${ }^{55}$ P. A. Bruckman de Renstrom, ${ }^{39}$ D. Bruncko, ${ }^{145 b}$ R. Bruneliere, ${ }^{48}$ S. Brunet,${ }^{60}$ A. Bruni, ${ }^{20 \mathrm{a}} \mathrm{G}$. Bruni, ${ }^{20 \mathrm{a}} \mathrm{M}$. Bruschi, ${ }^{20 \mathrm{a}}$ L. Bryngemark, ${ }^{80}$ T. Buanes,${ }^{14}$ Q. Buat, ${ }^{55} \mathrm{~F}$. Bucci,${ }^{49}$ P. Buchholz, ${ }^{142}$ R. M. Buckingham, ${ }^{119}$ A. G. Buckley, ${ }^{53}$ S. I. Buda, ${ }^{26 a}$ I. A. Budagov, ${ }^{64}$ B. Budick, ${ }^{109}$ F. Buehrer, ${ }^{48}$ L. Bugge, ${ }^{118}$ M. K. Bugge, ${ }^{118}$ O. Bulekov ${ }^{97}$ A. C. Bundock,${ }^{73}$ M. Bunse, ${ }^{43}$ H. Burckhart ${ }^{30}$ S. Burdin, ${ }^{73}$ B. Burghgrave,${ }^{107}$ S. Burke, ${ }^{130}$ I. Burmeister, ${ }^{43}$ E. Busato, ${ }^{34}$ V. Büscher, ${ }^{82}$ P. Bussey,${ }^{53}$ C. P. Buszello, ${ }^{167}$ B. Butler,${ }^{57}$ J. M. Butler, ${ }^{22}$ A. I. Butt, ${ }^{3}$ C. M. Buttar, ${ }^{53}$ J. M. Butterworth,${ }^{77}$ W. Buttinger, ${ }^{28}$ A. Buzatu, ${ }^{53}$ M. Byszewski, ${ }^{10}$ S. Cabrera Urbán, ${ }^{168}$ D. Caforio, ${ }^{20 a, 20 b}$ O. Cakir, ${ }^{4 a}$ P. Calafiura, ${ }^{15}$ G. Calderini, ${ }^{79}$ P. Calfayan, ${ }^{99}$ R. Calkins, ${ }^{107}$ L. P. Caloba,${ }^{24 a}$ R. Caloi, ${ }^{133 a, 133 b}$ D. Calvet,${ }^{34}$ S. Calvet ${ }^{34}$ R. Camacho Toro, ${ }^{49}$ P. Camarri,${ }^{134 a, 134 b}$ D. Cameron, ${ }^{118}$ L. M. Caminada, ${ }^{15}$ R. Caminal Armadans, ${ }^{12}$ S. Campana,${ }^{30}$ M. Campanelli, ${ }^{77}$ V. Canale, ${ }^{103 a, 103 b}$ F. Canelli, ${ }^{31}$ A. Canepa, ${ }^{160 a}$ J. Cantero, ${ }^{81}$ R. Cantrill, ${ }^{76}$ T. Cao, ${ }^{40}$ M. D. M. Capeans Garrido, ${ }^{30}$ I. Caprini, ${ }^{26 a}$ M. Caprini, ${ }^{26 a}$ M. Capua, ${ }^{37 a, 37 b}$ R. Caputo, ${ }^{82}$ R. Cardarelli, ${ }^{134 a}$ T. Carli, ${ }^{30}$ 
G. Carlino, ${ }^{103 a}$ L. Carminati, ${ }^{90 a, 90 b}$ S. Caron, ${ }^{105}$ E. Carquin, ${ }^{32 a}$ G. D. Carrillo-Montoya, ${ }^{146 c}$ A. A. Carter, ${ }^{75}$ J. R. Carter, ${ }^{28}$ J. Carvalho, ${ }^{125 a, 125 \mathrm{c}}$ D. Casadei, ${ }^{77}$ M. P. Casado, ${ }^{12}$ C. Caso, ${ }^{50 a, 50 b, a}$ E. Castaneda-Miranda, ${ }^{146 b}$ A. Castelli, ${ }^{106}$

V. Castillo Gimenez, ${ }^{168}$ N. F. Castro, ${ }^{125 a}$ P. Catastini, ${ }^{57}$ A. Catinaccio, ${ }^{30}$ J. R. Catmore, ${ }^{71}$ A. Cattai, ${ }^{30}$ G. Cattani, ${ }^{134 a, 134 b}$ S. Caughron, ${ }^{89}$ V. Cavaliere, ${ }^{166}$ D. Cavalli, ${ }^{90 a}$ M. Cavalli-Sforza, ${ }^{12}$ V. Cavasinni, ${ }^{123 a, 123 b}$ F. Ceradini, ${ }^{135 a, 135 b}$ B. Cerio, ${ }^{45}$ K. Cerny, ${ }^{128}$ A. S. Cerqueira, ${ }^{24 b}$ A. Cerri, ${ }^{150}$ L. Cerrito, ${ }^{75}$ F. Cerutti, ${ }^{15}$ M. Cerv,${ }^{30}$ A. Cervelli, ${ }^{17}$ S. A. Cetin, ${ }^{19 b}$ A. Chafaq, ${ }^{136 a}$ D. Chakraborty, ${ }^{107}$ I. Chalupkova, ${ }^{128}$ K. Chan, ${ }^{3}$ P. Chang, ${ }^{166}$ B. Chapleau, ${ }^{86}$ J. D. Chapman, ${ }^{28}$ D. Charfeddine,${ }^{116}$ D. G. Charlton, ${ }^{18}$ V. Chavda,${ }^{83}$ C. A. Chavez Barajas, ${ }^{30}$ S. Cheatham, ${ }^{86}$ S. Chekanov, ${ }^{6}$ S. V. Chekulaev, ${ }^{160 a}$ G. A. Chelkov, ${ }^{64}$ M. A. Chelstowska, ${ }^{88}$ C. Chen, ${ }^{63}$ H. Chen, ${ }^{25}$ K. Chen, ${ }^{149}$ L. Chen, ${ }^{33 d, h}$ S. Chen, ${ }^{33 \mathrm{c}}$ X. Chen, ${ }^{146 \mathrm{c}}$ Y. Chen, ${ }^{35}$ Y. Cheng, ${ }^{31}$ A. Cheplakov, ${ }^{64}$ R. Cherkaoui El Moursli, ${ }^{136 e}$ V. Chernyatin, ${ }^{25, a}$ E. Cheu, ${ }^{7}$ L. Chevalier, ${ }^{137}$ V. Chiarella, ${ }^{47}$ G. Chiefari, ${ }^{103 a, 103 b}$ J. T. Childers, ${ }^{30}$ A. Chilingarov, ${ }^{71}$ G. Chiodini, ${ }^{72 a}$ A. S. Chisholm, ${ }^{18}$ R. T. Chislett, ${ }^{77}$ A. Chitan, ${ }^{26 a}$ M. V. Chizhov, ${ }^{64}$ S. Chouridou, ${ }^{9}$ B. K. B. Chow, ${ }^{99}$ I. A. Christidi, ${ }^{77}$ D. Chromek-Burckhart, ${ }^{30}$ M. L. Chu, ${ }^{152}$ J. Chudoba, ${ }^{126}$ G. Ciapetti, ${ }^{133 a, 133 b}$ A. K. Ciftci, ${ }^{4 a}$ R. Ciftci, ${ }^{4 a}$ D. Cinca, ${ }^{62}$ V. Cindro, ${ }^{74}$ A. Ciocio,,${ }^{15}$ M. Cirilli, ${ }^{88}$ P. Cirkovic, ${ }^{13 b}$ Z. H. Citron, ${ }^{173}$ M. Citterio, ${ }^{90 a}$ M. Ciubancan, ${ }^{26 a}$ A. Clark, ${ }^{49}$ P. J. Clark, ${ }^{46}$ R. N. Clarke, ${ }^{15}$ W. Cleland, ${ }^{124}$ J. C. Clemens, ${ }^{84}$ B. Clement, ${ }^{55}$ C. Clement, ${ }^{147 a, 147 b}$ Y. Coadou, ${ }^{84}$ M. Cobal, ${ }^{165 a, 165 c}$ A. Coccaro, ${ }^{139}$ J. Cochran, ${ }^{63}$ L. Coffey, ${ }^{23}$ J. G. Cogan, ${ }^{144}$ J. Coggeshall, ${ }^{166}$ J. Colas, ${ }^{5}$ B. Cole,${ }^{35}$ S. Cole, ${ }^{107}$ A. P. Colijn, ${ }^{106}$ C. Collins-Tooth,${ }^{53}$ J. Collot, ${ }^{55}$ T. Colombo,${ }^{58 c}$ G. Colon, ${ }^{85}$ G. Compostella, ${ }^{100}$ P. Conde Muiño, ${ }^{125 a, 125 b}$ E. Coniavitis, ${ }^{167}$ M. C. Conidi, ${ }^{12}$ I. A. Connelly, ${ }^{76}$ S. M. Consonni, ${ }^{90 a, 90 b}$ V. Consorti, ${ }^{48}$ S. Constantinescu, ${ }^{26 a}$ C. Conta, ${ }^{120 a, 120 b}$ G. Conti, ${ }^{57}$ F. Conventi, ${ }^{103 a, i}$ M. Cooke ${ }^{15}$ B. D. Cooper ${ }^{77}$ A. M. Cooper-Sarkar, ${ }^{119}$ N. J. Cooper-Smith, ${ }^{76}$ K. Copic, ${ }^{15}$ T. Cornelissen, ${ }^{176}$ M. Corradi, ${ }^{20 a}$ F. Corriveau,,${ }^{86, j}$ A. Corso-Radu, ${ }^{164}$ A. Cortes-Gonzalez, ${ }^{12}$ G. Cortiana, ${ }^{100}$ G. Costa, ${ }^{90 a}$ M. J. Costa, ${ }^{168}$ D. Costanzo, ${ }^{140}$ D. Côté, ${ }^{8}$ G. Cottin, ${ }^{32 a}$ G. Cowan, ${ }^{76}$ B. E. Cox,${ }^{83}$ K. Cranmer,${ }^{109}$ G. Cree, ${ }^{29}$ S. Crépé-Renaudin, ${ }^{55}$ F. Crescioli, ${ }^{79}$ M. Crispin Ortuzar, ${ }^{119}$ M. Cristinziani, ${ }^{21}$ G. Crosetti, ${ }^{37 a, 37 b}$ C.-M. Cuciuc ${ }^{26 a}$ C. Cuenca Almenar, ${ }^{177}$ T. Cuhadar Donszelmann, ${ }^{140}$ J. Cummings, ${ }^{177}$ M. Curatolo, ${ }^{47}$ C. Cuthbert, ${ }^{151}$ H. Czirr, ${ }^{142}$ P. Czodrowski, ${ }^{3}$ Z. Czyczula, ${ }^{177}$ S. D’Auria, ${ }^{53}$ M. D’Onofrio, ${ }^{73}$ A. D’Orazio, ${ }^{133 a, 133 b}$ M. J. Da Cunha Sargedas De Sousa, ${ }^{125 a, 125 b}$ C. Da Via ${ }^{83}$ W. Dabrowski, ${ }^{38 a}$ A. Dafinca, ${ }^{119}$ T. Dai ${ }^{88}$ F. Dallaire, ${ }^{94}$ C. Dallapiccola, ${ }^{85}$ M. Dam,${ }^{36}$ A. C. Daniells, ${ }^{18}$ M. Dano Hoffmann, ${ }^{36}$ V. Dao, ${ }^{105}$ G. Darbo, ${ }^{50 a}$ G. L. Darlea, ${ }^{26 c}$ S. Darmora, ${ }^{8}$ J. A. Dassoulas, ${ }^{42}$ W. Davey, ${ }^{21}$ C. David, ${ }^{170}$ T. Davidek, ${ }^{128}$ E. Davies, ${ }^{119, d}$ M. Davies, ${ }^{94}$ O. Davignon, ${ }^{79}$ A. R. Davison, ${ }^{77}$ Y. Davygora, ${ }^{58 a}$ E. Dawe, ${ }^{143}$ I. Dawson, ${ }^{140}$ R. K. Daya-Ishmukhametova, ${ }^{23}$ K. De,${ }^{8}$ R. de Asmundis, ${ }^{103 a}$ S. De Castro, ${ }^{20 a, 20 b}$ S. De Cecco, ${ }^{79}$ J. de Graat, ${ }^{99}$ N. De Groot ${ }^{105}$ P. de Jong, ${ }^{106}$ C. De La Taille, ${ }^{116}$ H. De la Torre, ${ }^{81}$ F. De Lorenzi ${ }^{63}$ L. De Nooij, ${ }^{106}$ D. De Pedis, ${ }^{133 a}$ A. De Salvo, ${ }^{133 a}$ U. De Sanctis,${ }^{165 a, 165 c}$ A. De Santo, ${ }^{150}$

J. B. De Vivie De Regie, ${ }^{116}$ G. De Zorzi, ${ }^{133 a, 133 b}$ W. J. Dearnaley, ${ }^{71}$ R. Debbe,${ }^{25}$ C. Debenedetti, ${ }^{46}$ B. Dechenaux, ${ }^{55}$ D. V. Dedovich, ${ }^{64}$ J. Degenhardt, ${ }^{121}$ I. Deigaard, ${ }^{106}$ J. Del Peso, ${ }^{81}$ T. Del Prete, ${ }^{123 a, 123 b}$ T. Delemontex,${ }^{55}$ F. Deliot, ${ }^{137}$ M. Deliyergiyev, ${ }^{74}$ A. Dell'Acqua ${ }^{30}$ L. Dell'Asta ${ }^{22}$ M. Della Pietra, ${ }^{103 a, i}$ D. della Volpe,${ }^{49}$ M. Delmastro, ${ }^{5}$ P. A. Delsart,${ }^{55}$ C. Deluca, ${ }^{106}$ S. Demers,${ }^{177}$ M. Demichev,${ }^{64}$ A. Demilly, ${ }^{79}$ B. Demirkoz, ${ }^{12, k}$ S. P. Denisov, ${ }^{129}$ D. Derendarz,${ }^{39}$ J. E. Derkaoui, ${ }^{136 d}$ F. Derue, ${ }^{79}$ P. Dervan, ${ }^{73}$ K. Desch,${ }^{21}$ P. O. Deviveiros,${ }^{106}$ A. Dewhurst, ${ }^{130}$ S. Dhaliwal, ${ }^{106}$ A. Di Ciaccio, ${ }^{134 a, 134 b}$ L. Di Ciaccio,${ }^{5}$ A. Di Domenico, ${ }^{133 a, 133 b}$ C. Di Donato, ${ }^{103 a, 103 b}$ A. Di Girolamo, ${ }^{30}$ B. Di Girolamo, ${ }^{30}$ A. Di Mattia, ${ }^{153}$ B. Di Micco, ${ }^{135 a, 135 b}$ R. Di Nardo, ${ }^{47}$ A. Di Simone, ${ }^{48}$ R. Di Sipio, ${ }^{20 a, 20 b}$ D. Di Valentino, ${ }^{29}$ M. A. Diaz, ${ }^{32 a}$ E. B. Diehl,${ }^{88}$ J. Dietrich, ${ }^{42}$ T. A. Dietzsch, ${ }^{58 a}$ S. Diglio, ${ }^{87}$ A. Dimitrievska, ${ }^{13 a}$ K. Dindar Yagci, ${ }^{40}$ J. Dingfelder, ${ }^{21}$ C. Dionisi, ${ }^{133 a, 133 b}$ P. Dita, ${ }^{26 a}$ S. Dita, ${ }^{26 a}$ F. Dittus,${ }^{30}$ F. Djama,${ }^{84}$ T. Djobava, ${ }^{51 b}$ M. A. B. do Vale, ${ }^{24 c}$

A. Do Valle Wemans, ${ }^{125 a, 125 g}$ T. K. O. Doan, ${ }^{5}$ D. Dobos,${ }^{30}$ E. Dobson, ${ }^{77}$ J. Dodd,${ }^{35}$ C. Doglioni, ${ }^{49}$ T. Doherty,${ }^{53}$ T. Dohmae, ${ }^{156}$ J. Dolejsi, ${ }^{128}$ Z. Dolezal, ${ }^{128}$ B. A. Dolgoshein, ${ }^{97, a}$ M. Donadelli, ${ }^{24 d}$ S. Donati, ${ }^{123 a, 123 b}$ P. Dondero, ${ }^{120 a, 120 b}$ J. Donini, ${ }^{34}$ J. Dopke, ${ }^{30}$ A. Doria, ${ }^{103 a}$ A. Dos Anjos, ${ }^{174}$ A. Dotti, ${ }^{123 a, 123 b}$ M. T. Dova,${ }^{70}$ A. T. Doyle,${ }^{53}$ M. Dris,${ }^{10}$ J. Dubbert ${ }^{88}$ S. Dube, ${ }^{15}$ E. Dubreuil, ${ }^{34}$ E. Duchovni, ${ }^{173}$ G. Duckeck, ${ }^{99}$ O. A. Ducu, ${ }^{26 a}$ D. Duda, ${ }^{176}$ A. Dudarev, ${ }^{30}$ F. Dudziak, ${ }^{63}$ L. Duflot, ${ }^{116}$ L. Duguid, ${ }^{76}$ M. Dührssen, ${ }^{30}$ M. Dunford, ${ }^{58 a}$ H. Duran Yildiz, ${ }^{4 a}$ M. Düren,${ }^{52}$ M. Dwuznik,${ }^{38 a}$ J. Ebke, ${ }^{99}$ W. Edson, ${ }^{2}$ C. A. Edwards, ${ }^{76}$ N. C. Edwards ${ }^{46}$ W. Ehrenfeld,${ }^{21}$ T. Eifert, ${ }^{144}$ G. Eigen, ${ }^{14}$ K. Einsweiler, ${ }^{15}$ T. Ekelof,,${ }^{167}$

M. El Kacimi, ${ }^{136 c}$ M. Ellert, ${ }^{167}$ S. Elles,${ }^{5}$ F. Ellinghaus, ${ }^{82}$ K. Ellis,${ }^{75}$ N. Ellis, ${ }^{30}$ J. Elmsheuser, ${ }^{99}$ M. Elsing, ${ }^{30}$ D. Emeliyanov ${ }^{130}$ Y. Enari, ${ }^{156}$ O. C. Endner, ${ }^{82}$ M. Endo, ${ }^{117}$ R. Engelmann, ${ }^{149}$ J. Erdmann, ${ }^{177}$ A. Ereditato, ${ }^{17}$ D. Eriksson, ${ }^{147 a}$ G. Ernis, ${ }^{176}$ J. Ernst, ${ }^{2}$ M. Ernst, ${ }^{25}$ J. Ernwein, ${ }^{137}$ D. Errede, ${ }^{166}$ S. Errede, ${ }^{166}$ E. Ertel, ${ }^{82}$ M. Escalier, ${ }^{116}$ H. Esch, ${ }^{43}$ C. Escobar, ${ }^{124}$ X. Espinal Curull, ${ }^{12}$ B. Esposito, ${ }^{47}$ F. Etienne, ${ }^{84}$ A. I. Etienvre, ${ }^{137}$ E. Etzion, ${ }^{154}$ D. Evangelakou,${ }^{54}$ H. Evans,${ }^{60}$ L. Fabbri, ${ }^{20 \mathrm{a}, 20 \mathrm{~b}}$ G. Facini, ${ }^{30}$ R. M. Fakhrutdinov, ${ }^{129}$ S. Falciano, ${ }^{133 \mathrm{a}}$ Y. Fang, ${ }^{33 \mathrm{a}}$ M. Fanti, ${ }^{90 \mathrm{a}, 90 \mathrm{~b}}$ A. Farbin, ${ }^{8}$ A. Farilla, ${ }^{135 \mathrm{a}}$ T. Farooque, ${ }^{12}$ S. Farrell, ${ }^{164}$ S. M. Farrington, ${ }^{171}$ P. Farthouat, ${ }^{30}$ F. Fassi, ${ }^{168}$ P. Fassnacht, ${ }^{30}$ D. Fassouliotis, ${ }^{9}$ 
B. Fatholahzadeh, ${ }^{159}$ A. Favareto, ${ }^{50,50 \mathrm{~b}}$ L. Fayard, ${ }^{116}$ P. Federic, ${ }^{145 \mathrm{a}}$ O. L. Fedin, ${ }^{122}$ W. Fedorko, ${ }^{169}$ M. Fehling-Kaschek,${ }^{48}$ S. Feigl,${ }^{30}$ L. Feligioni,${ }^{84}$ C. Feng, ${ }^{33 d}$ E. J. Feng, ${ }^{6}$ H. Feng, ${ }^{88}$ A. B. Fenyuk, ${ }^{129}$ W. Fernando, ${ }^{6}$ S. Ferrag,${ }^{53}$ J. Ferrando, ${ }^{53}$ V. Ferrara, ${ }^{42}$ A. Ferrari, ${ }^{167}$ P. Ferrari, ${ }^{106}$ R. Ferrari, ${ }^{120 a}$ D. E. Ferreira de Lima, ${ }^{53}$ A. Ferrer, ${ }^{168}$ D. Ferrere, ${ }^{49}$ C. Ferretti ${ }^{88}$ A. Ferretto Parodi ${ }^{50 a, 50 b}$ M. Fiascaris, ${ }^{31}$ F. Fiedler, ${ }^{82}$ A. Filipčič, ${ }^{74}$ M. Filipuzzi, ${ }^{42}$ F. Filthaut, ${ }^{105}$ M. Fincke-Keeler,${ }^{170}$ K. D. Finelli, ${ }^{45}$ M. C. N. Fiolhais, ${ }^{125 a, 125 c, 1}$ L. Fiorini, ${ }^{168}$ A. Firan, ${ }^{40}$ J. Fischer, ${ }^{176}$ M. J. Fisher,${ }^{110}$ E. A. Fitzgerald, ${ }^{23}$ M. Flechl ${ }^{48}$ I. Fleck,${ }^{142}$ P. Fleischmann, ${ }^{175}$ S. Fleischmann, ${ }^{176}$ G. T. Fletcher ${ }^{140}$ G. Fletcher, ${ }^{75}$ T. Flick, ${ }^{176}$ A. Floderus,${ }^{80}$ L. R. Flores Castillo, ${ }^{174}$ A. C. Florez Bustos, ${ }^{160 b}$ M. J. Flowerdew, ${ }^{100}$ A. Formica, ${ }^{137}$ A. Forti, ${ }^{83}$ D. Fortin,${ }^{160 a}$ D. Fournier, ${ }^{116}$ H. Fox ${ }^{71}$ P. Francavilla, ${ }^{12}$ M. Franchini, ${ }^{20 a, 20 b}$ S. Franchino, ${ }^{30}$ D. Francis,${ }^{30}$ M. Franklin,${ }^{57}$ S. Franz,${ }^{61}$ M. Fraternali, ${ }^{120 a, 120 b}$ S. Fratina, ${ }^{121}$ S. T. French, ${ }^{28}$ C. Friedrich, ${ }^{42}$ F. Friedrich, ${ }^{44}$ D. Froidevaux,${ }^{30}$ J. A. Frost,${ }^{28}$ C. Fukunaga, ${ }^{157}$ E. Fullana Torregrosa, ${ }^{128}$ B. G. Fulsom, ${ }^{144}$ J. Fuster, ${ }^{168}$ C. Gabaldon, ${ }^{55}$ O. Gabizon, ${ }^{173}$ A. Gabrielli, ${ }^{20 a, 20 b}$ A. Gabrielli, ${ }^{133 a, 133 b}$ S. Gadatsch, ${ }^{106}$ T. Gadfort, ${ }^{25}$ S. Gadomski, ${ }^{49}$ G. Gagliardi, ${ }^{50,50 b}$ P. Gagnon, ${ }^{60}$ C. Galea, ${ }^{99}$ B. Galhardo, ${ }^{125 a, 125 c}$ E. J. Gallas, ${ }^{119}$ V. Gallo, ${ }^{17}$ B. J. Gallop, ${ }^{130}$ P. Gallus, ${ }^{127}$ G. Galster, ${ }^{36}$ K. K. Gan,${ }^{110}$ R. P. Gandrajula ${ }^{62}$ J. Gao, ${ }^{33 b, h}$ Y. S. Gao, ${ }^{144, f}$ F. M. Garay Walls, ${ }^{46}$ F. Garberson, ${ }^{177}$ C. García, ${ }^{168}$ J. E. García Navarro, ${ }^{168}$ M. Garcia-Sciveres,${ }^{15}$ R. W. Gardner, ${ }^{31}$ N. Garelli, ${ }^{144}$ V. Garonne, ${ }^{30}$ C. Gatti, ${ }^{47}$ G. Gaudio, ${ }^{120 a}$ B. Gaur, ${ }^{142}$ L. Gauthier, ${ }^{94}$ P. Gauzzi, ${ }^{133 a, 133 b}$ I. L. Gavrilenko, ${ }^{95}$ C. Gay, ${ }^{169}$ G. Gaycken, ${ }^{21}$ E. N. Gazis, ${ }^{10}$ P. Ge, ${ }^{33 d, m}$ Z. Gecse, ${ }^{169}$ C. N. P. Gee, ${ }^{130}$ D. A. A. Geerts, ${ }^{106}$ Ch. Geich-Gimbel, ${ }^{21}$ K. Gellerstedt, ${ }^{147 a, 147 b}$ C. Gemme, ${ }^{50 \mathrm{a}}$ A. Gemmell, ${ }^{53}$ M. H. Genest, ${ }^{55}$ S. Gentile, ${ }^{133 a, 133 b}$ M. George ${ }^{54}$

S. George ${ }^{76}$ D. Gerbaudo, ${ }^{164}$ A. Gershon, ${ }^{154}$ H. Ghazlane,${ }^{136 b}$ N. Ghodbane, ${ }^{34}$ B. Giacobbe,${ }^{20 a}$ S. Giagu, ${ }^{133 a, 133 b}$ V. Giangiobbe, ${ }^{12}$ P. Giannetti, ${ }^{123 a, 123 b}$ F. Gianotti, ${ }^{30}$ B. Gibbard, ${ }^{25}$ S. M. Gibson, ${ }^{76}$ M. Gilchriese, ${ }^{15}$ T. P. S. Gillam, ${ }^{28}$ D. Gillberg, ${ }^{30}$ A. R. Gillman, ${ }^{130}$ D. M. Gingrich,,${ }^{3, \mathrm{e}}$ N. Giokaris, ${ }^{9}$ M. P. Giordani, ${ }^{165 a, 165 \mathrm{c}}$ R. Giordano, ${ }^{103 a, 103 b}$ F. M. Giorgi, ${ }^{16}$ P. Giovannini, ${ }^{100}$ P. F. Giraud, ${ }^{137}$ D. Giugni, ${ }^{90 a}$ C. Giuliani, ${ }^{48}$ M. Giunta, ${ }^{94}$ B. K. Gjelsten, ${ }^{118}$ I. Gkialas,${ }^{155, n}$ L. K. Gladilin, ${ }^{98}$ C. Glasman, ${ }^{81}$ J. Glatzer, ${ }^{21}$ A. Glazov, ${ }^{42}$ G. L. Glonti, ${ }^{64}$ M. Goblirsch-Kolb, ${ }^{100}$ J. R. Goddard ${ }^{75}$ J. Godfrey, ${ }^{143}$ J. Godlewski, ${ }^{30}$ C. Goeringer, ${ }^{82}$ S. Goldfarb ${ }^{88}$ T. Golling ${ }^{177}$ D. Golubkov, ${ }^{129}$ A. Gomes, ${ }^{125 a, 125 b, 125 \mathrm{~d}}$ L. S. Gomez Fajardo ${ }^{42}$ R. Gonçalo, ${ }^{76}$ J. Goncalves Pinto Firmino Da Costa, ${ }^{42}$ L. Gonella, ${ }^{21}$ S. González de la Hoz, ${ }^{168}$ G. Gonzalez Parra, ${ }^{12}$ M. L. Gonzalez Silva, ${ }^{27}$ S. Gonzalez-Sevilla, ${ }^{49}$ L. Goossens, ${ }^{30}$ P. A. Gorbounov, ${ }^{96}$ H. A. Gordon, ${ }^{25}$ I. Gorelov, ${ }^{104}$ G. Gorfine, ${ }^{176}$ B. Gorini, ${ }^{30}$ E. Gorini $,{ }^{72 a}, 72 b$ A. Gorišek,${ }^{74}$ E. Gornicki, ${ }^{39}$ A. T. Goshaw, ${ }^{6}$ C. Gössling, ${ }^{43}$ M. I. Gostkin ${ }^{64}$ M. Gouighri, ${ }^{136 a}$ D. Goujdami, ${ }^{136 c}$ M. P. Goulette, ${ }^{49}$ A. G. Goussiou, ${ }^{139}$ C. Goy, ${ }^{5}$ S. Gozpinar, ${ }^{23}$ H. M. X. Grabas, ${ }^{137}$ L. Graber, ${ }^{54}$ I. Grabowska-Bold, ${ }^{38 a}$ P. Grafström, ${ }^{20 a, 20 b}$ K-J. Grahn, ${ }^{42}$ J. Gramling, ${ }^{49}$ E. Gramstad, ${ }^{118}$ F. Grancagnolo,${ }^{72 a}$ S. Grancagnolo, ${ }^{16}$ V. Grassi, ${ }^{149}$ V. Gratchev, ${ }^{122}$ H. M. Gray, ${ }^{30}$ J. A. Gray, ${ }^{149}$ E. Graziani, ${ }^{135 a}$ O. G. Grebenyuk, ${ }^{122}$ Z. D. Greenwood, ${ }^{78,0}$ K. Gregersen, ${ }^{36}$ I. M. Gregor, ${ }^{42}$ P. Grenier, ${ }^{144}$ J. Griffiths, ${ }^{8}$ N. Grigalashvili, ${ }^{64}$ A. A. Grillo, ${ }^{138}$ K. Grimm, ${ }^{71}$ S. Grinstein, ${ }^{12, p}$ Ph. Gris ${ }^{34}$ Y. V. Grishkevich, ${ }^{98}$ J.-F. Grivaz, ${ }^{116}$ J. P. Grohs, ${ }^{44}$ A. Grohsjean, ${ }^{42}$ E. Gross, ${ }^{173}$ J. Grosse-Knetter, ${ }^{54}$ G. C. Grossi, ${ }^{134 a, 134 b}$ J. Groth-Jensen, ${ }^{173}$ Z. J. Grout, ${ }^{150}$ K. Grybel, ${ }^{142}$ L. Guan, ${ }^{33 b}$ F. Guescini, ${ }^{49}$ D. Guest, ${ }^{177}$ O. Gueta, ${ }^{154}$ C. Guicheney, ${ }^{34}$ E. Guido, ${ }^{50 a, 50 b}$ T. Guillemin, ${ }^{116}$ S. Guindon, ${ }^{2}$ U. Gul, ${ }^{53}$ C. Gumpert, ${ }^{44}$ J. Gunther, ${ }^{127}$ J. Guo, ${ }^{35}$ S. Gupta, ${ }^{119}$ P. Gutierrez, ${ }^{112}$ N. G. Gutierrez Ortiz,${ }^{53}$ C. Gutschow, ${ }^{77}$ N. Guttman, ${ }^{154}$ C. Guyot, ${ }^{137}$ C. Gwenlan, ${ }^{119}$ C. B. Gwilliam, ${ }^{73}$ A. Haas, ${ }^{109}$ C. Haber ${ }^{15}$ H. K. Hadavand,${ }^{8}$ P. Haefner, ${ }^{21}$ S. Hageboeck,${ }^{21}$ Z. Hajduk,${ }^{39}$ H. Hakobyan, ${ }^{178}$ M. Haleem, ${ }^{42}$ D. Hall, ${ }^{119}$ G. Halladjian, ${ }^{89}$ K. Hamacher, ${ }^{176}$ P. Hamal, ${ }^{114}$ K. Hamano,${ }^{87}$ M. Hamer, ${ }^{54}$ A. Hamilton, ${ }^{146 a, q}$ S. Hamilton, ${ }^{162}$ L. Han, ${ }^{33 b}$ K. Hanagaki, ${ }^{117}$ K. Hanawa, ${ }^{156}$ M. Hance, ${ }^{15}$ P. Hanke, ${ }^{58 a}$ J. R. Hansen, ${ }^{36}$ J. B. Hansen, ${ }^{36}$ J. D. Hansen, ${ }^{36}$ P. H. Hansen, ${ }^{36}$ P. Hansson, ${ }^{144}$ K. Hara, ${ }^{161}$ A. S. Hard ${ }^{174}$ T. Harenberg, ${ }^{176}$ S. Harkusha, ${ }^{91}$

D. Harper ${ }^{88}$ R. D. Harrington, ${ }^{46}$ O. M. Harris, ${ }^{139}$ P. F. Harrison, ${ }^{171}$ F. Hartjes, ${ }^{106}$ A. Harvey,${ }^{56}$ S. Hasegawa, ${ }^{102}$

Y. Hasegawa, ${ }^{141}$ S. Hassani, ${ }^{137}$ S. Haug, ${ }^{17}$ M. Hauschild ${ }^{30}$ R. Hauser ${ }^{89}$ M. Havranek,${ }^{21}$ C. M. Hawkes, ${ }^{18}$ R. J. Hawkings,${ }^{30}$ A. D. Hawkins, ${ }^{80}$ T. Hayashi, ${ }^{161}$ D. Hayden, ${ }^{89}$ C. P. Hays, ${ }^{119}$ H. S. Hayward, ${ }^{73}$ S. J. Haywood, ${ }^{130}$ S. J. Head, ${ }^{18}$ T. Heck, ${ }^{82}$ V. Hedberg, ${ }^{80}$ L. Heelan, ${ }^{8}$ S. Heim, ${ }^{121}$ T. Heim, ${ }^{176}$ B. Heinemann, ${ }^{15}$ S. Heisterkamp, ${ }^{36}$ J. Hejbal, ${ }^{126}$ L. Helary, ${ }^{22}$ C. Heller ${ }^{99}$ M. Heller, ${ }^{30}$ S. Hellman, ${ }^{147 a, 147 b}$ D. Hellmich, ${ }^{21}$ C. Helsens, ${ }^{30}$ J. Henderson, ${ }^{119}$ R. C. W. Henderson, ${ }^{71}$ A. Henrichs, ${ }^{177}$ A. M. Henriques Correia ${ }^{30}$ S. Henrot-Versille, ${ }^{116}$ C. Hensel,${ }^{54}$ G. H. Herbert, ${ }^{16}$ Y. Hernández Jiménez, ${ }^{168}$ R. Herrberg-Schubert, ${ }^{16}$ G. Herten ${ }^{48}$ R. Hertenberger, ${ }^{99}$ L. Hervas,${ }^{30}$ G. G. Hesketh ${ }^{77}$ N. P. Hessey, ${ }^{106}$ R. Hickling, ${ }^{75}$ E. Higón-Rodriguez, ${ }^{168}$ J. C. Hill, ${ }^{28}$ K. H. Hiller, ${ }^{42}$ S. Hillert, ${ }^{21}$ S. J. Hillier, ${ }^{18}$ I. Hinchliffe, ${ }^{15}$ E. Hines, ${ }^{121}$ M. Hirose, ${ }^{117}$ D. Hirschbuehl, ${ }^{176}$ J. Hobbs, ${ }^{149}$ N. Hod,${ }^{106}$ M. C. Hodgkinson, ${ }^{140}$ P. Hodgson, ${ }^{140}$ A. Hoecker,${ }^{30}$ M. R. Hoeferkamp, ${ }^{104}$ J. Hoffman, ${ }^{40}$ D. Hoffmann, ${ }^{84}$ J. I. Hofmann, ${ }^{58 a}$ M. Hohlfeld,${ }^{82}$ T. R. Holmes, ${ }^{15}$ T. M. Hong, ${ }^{121}$ L. Hooft van Huysduynen, ${ }^{109}$ J-Y. Hostachy, ${ }^{55}$ S. Hou, ${ }^{152}$ A. Hoummada, ${ }^{136 a}$ J. Howard, ${ }^{119}$ J. Howarth ${ }^{83}$ M. Hrabovsky, ${ }^{114}$ I. Hristova, ${ }^{16}$ J. Hrivnac, ${ }^{116}$ T. Hryn'ova, ${ }^{5}$ P. J. Hsu, ${ }^{82}$ S.-C. Hsu, ${ }^{139}$ D. Hu, ${ }^{35}$ X. Hu, ${ }^{25}$ Y. Huang, ${ }^{146 c}$ Z. Hubacek, ${ }^{30}$ F. Hubaut,${ }^{84}$ F. Huegging, ${ }^{21}$ 
A. Huettmann, ${ }^{42}$ T. B. Huffman, ${ }^{119}$ E. W. Hughes,${ }^{35}$ G. Hughes,${ }^{71}$ M. Huhtinen, ${ }^{30}$ T. A. Hülsing, ${ }^{82}$ M. Hurwitz, ${ }^{15}$ N. Huseynov, ${ }^{64, c}$ J. Huston, ${ }^{89}$ J. Huth,${ }^{57}$ G. Iacobucci, ${ }^{49}$ G. Iakovidis, ${ }^{10}$ I. Ibragimov, ${ }^{142}$ L. Iconomidou-Fayard, ${ }^{116}$ J. Idarraga, ${ }^{116}$ E. Ideal, ${ }^{177}$ P. Iengo, ${ }^{103 a}$ O. Igonkina, ${ }^{106}$ T. Iizawa, ${ }^{172}$ Y. Ikegami,${ }^{65} \mathrm{~K}$. Ikematsu, ${ }^{142} \mathrm{M}$. Ikeno, ${ }^{65} \mathrm{D}$. Iliadis, ${ }^{155}$ N. Ilic, ${ }^{159}$ Y. Inamaru, ${ }^{66}$ T. Ince, ${ }^{100}$ P. Ioannou, ${ }^{9}$ M. Iodice, ${ }^{135 a}$ K. Iordanidou, ${ }^{9}$ V. Ippolito, ${ }^{133 a, 133 b}$ A. Irles Quiles, ${ }^{168}$ C. Isaksson, ${ }^{167} \mathrm{M}$. Ishino, ${ }^{67} \mathrm{M}$. Ishitsuka, ${ }^{158} \mathrm{R}$. Ishmukhametov, ${ }^{110} \mathrm{C}$. Issever, ${ }^{119} \mathrm{~S}$. Istin, ${ }^{19 a} \mathrm{~A}$. V. Ivashin, ${ }^{129} \mathrm{~W}$. Iwanski, ${ }^{39}$ H. Iwasaki, ${ }^{65}$ J. M. Izen, ${ }^{41}$ V. Izzo, ${ }^{103 a}$ B. Jackson, ${ }^{121}$ J. N. Jackson, ${ }^{73}$ M. Jackson, ${ }^{73}$ P. Jackson, ${ }^{1}$ M. R. Jaekel, ${ }^{30}$ V. Jain, ${ }^{2}$ K. Jakobs, ${ }^{48}$ S. Jakobsen, ${ }^{36}$ T. Jakoubek, ${ }^{126}$ J. Jakubek, ${ }^{127}$ D. O. Jamin,${ }^{152}$ D. K. Jana, ${ }^{78}$ E. Jansen, ${ }^{77}$ H. Jansen, ${ }^{30}$ J. Janssen, ${ }^{21}$ M. Janus, ${ }^{171}$ R. C. Jared, ${ }^{174}$ G. Jarlskog, ${ }^{80}$ L. Jeanty, ${ }^{15}$ G.-Y. Jeng, ${ }^{151}$ I. Jen-La Plante, ${ }^{31}$ D. Jennens, ${ }^{87}$ P. Jenni, ${ }^{48, \mathrm{r}}$ J. Jentzsch, ${ }^{43}$ C. Jeske, ${ }^{171}$ S. Jézéquel, ${ }^{5} \mathrm{M}$. K. Jha, ${ }^{20 \mathrm{a}} \mathrm{H}$. Ji, ${ }^{174} \mathrm{~W}$. Ji, ${ }^{82} \mathrm{~J}$. Jia, ${ }^{149}$ Y. Jiang, ${ }^{33 \mathrm{~b}}$

M. Jimenez Belenguer, ${ }^{42}$ S. Jin, ${ }^{33 a}$ A. Jinaru, ${ }^{26 a}$ O. Jinnouchi, ${ }^{158}$ M. D. Joergensen, ${ }^{36}$ D. Joffe, ${ }^{40}$ K. E. Johansson, ${ }^{147 a}$ P. Johansson, ${ }^{140}$ K. A. Johns, ${ }^{7}$ K. Jon-And, ${ }^{147 a, 147 b}$ G. Jones, ${ }^{171}$ R. W. L. Jones, ${ }^{71}$ T. J. Jones, ${ }^{73}$ P. M. Jorge, ${ }^{125 a, 125 b}$

K. D. Joshi, ${ }^{83}$ J. Jovicevic, ${ }^{148}$ X. Ju, ${ }^{174}$ C. A. Jung, ${ }^{43}$ R. M. Jungst ${ }^{30}$ P. Jussel,${ }^{61}$ A. Juste Rozas, ${ }^{12, p}$ M. Kaci, ${ }^{168}$ A. Kaczmarska, ${ }^{39}$ M. Kado, ${ }^{116}$ H. Kagan, ${ }^{110}$ M. Kagan, ${ }^{144}$ E. Kajomovitz, ${ }^{45}$ S. Kalinin, ${ }^{176}$ S. Kama, ${ }^{40}$ N. Kanaya, ${ }^{156}$ M. Kaneda, ${ }^{30}$ S. Kaneti, ${ }^{28}$ T. Kanno, ${ }^{158}$ V. A. Kantserov, ${ }^{97}$ J. Kanzaki, ${ }^{65}$ B. Kaplan, ${ }^{109}$ A. Kapliy, ${ }^{31}$ D. Kar, ${ }^{53}$ K. Karakostas, ${ }^{10}$ N. Karastathis, ${ }^{10}$ M. Karnevskiy, ${ }^{82}$ S. N. Karpov,${ }^{64}$ K. Karthik, ${ }^{109}$ V. Kartvelishvili, ${ }^{71}$ A. N. Karyukhin, ${ }^{129}$ L. Kashif, ${ }^{174}$ G. Kasieczka, ${ }^{58 b}$ R. D. Kass ${ }^{110}$ A. Kastanas, ${ }^{14}$ Y. Kataoka, ${ }^{156}$ A. Katre, ${ }^{49}$ J. Katzy, ${ }^{42}$ V. Kaushik, ${ }^{7}$ K. Kawagoe ${ }^{69}$ T. Kawamoto, ${ }^{156}$ G. Kawamura, ${ }^{54}$ S. Kazama, ${ }^{156}$ V. F. Kazanin, ${ }^{108}$ M. Y. Kazarinov, ${ }^{64}$ R. Keeler, ${ }^{170}$ P. T. Keener, ${ }^{121}$ R. Kehoe ${ }^{40}$ M. Keil,${ }^{54}$ J. S. Keller, ${ }^{139}$ H. Keoshkerian, ${ }^{5}$ O. Kepka, ${ }^{126}$ B. P. Kerševan, ${ }^{74}$ S. Kersten, ${ }^{176}$ K. Kessoku, ${ }^{156}$ J. Keung, ${ }^{159}$ F. Khalil-zada, ${ }^{11}$ H. Khandanyan, ${ }^{147 a, 147 b}$ A. Khanov, ${ }^{113}$ D. Kharchenko, ${ }^{64}$ A. Khodinov, ${ }^{97}$ A. Khomich, ${ }^{58 \mathrm{a}}$ T. J. Khoo, ${ }^{28}$ G. Khoriauli, ${ }^{21}$ A. Khoroshilov, ${ }^{176}$ V. Khovanskiy, ${ }^{96}$ E. Khramov, ${ }^{64}$ J. Khubua, ${ }^{51 b}$ H. Kim, ${ }^{147 a, 147 b}$ S. H. Kim, ${ }^{161}$ N. Kimura, ${ }^{172}$ O. Kind, ${ }^{16}$ B. T. King, ${ }^{73}$ M. King, ${ }^{168}$ R. S. B. King, ${ }^{119}$ S. B. King, ${ }^{169}$ J. Kirk, ${ }^{130}$ A. E. Kiryunin, ${ }^{100}$ T. Kishimoto ${ }^{66}$ D. Kisielewska, ${ }^{38 a}$ T. Kitamura, ${ }^{66}$ T. Kittelmann, ${ }^{124}$ K. Kiuchi, ${ }^{161}$ E. Kladiva, ${ }^{145 b}$ M. Klein, ${ }^{73}$ U. Klein, ${ }^{73}$ K. Kleinknecht ${ }^{82}$ P. Klimek, ${ }^{147 a, 147 b}$ A. Klimentov, ${ }^{25}$ R. Klingenberg, ${ }^{43}$ J. A. Klinger, ${ }^{83}$ E. B. Klinkby, ${ }^{36}$ T. Klioutchnikova, ${ }^{30}$ P. F. Klok, ${ }^{105}$ E.-E. Kluge, ${ }^{58 a}$ P. Kluit, ${ }^{106}$ S. Kluth, ${ }^{100}$ E. Kneringer, ${ }^{61}$ E.B.F.G. Knoops ${ }^{84}$ A. Knue ${ }^{53}$ T. Kobayashi, ${ }^{156}$ M. Kobel,${ }^{44}$ M. Kocian,${ }^{144}$ P. Kodys ${ }^{128}$ S. Koenig ${ }^{82}$ P. Koevesarki, ${ }^{21}$ T. Koffas, ${ }^{29}$ E. Koffeman, ${ }^{106}$ L. A. Kogan, ${ }^{119}$ S. Kohlmann, ${ }^{176}$ Z. Kohout, ${ }^{127}$ T. Kohriki, ${ }^{65}$ T. Koi, ${ }^{144}$ H. Kolanoski, ${ }^{16}$

I. Koletsou, ${ }^{5}$ J. Koll, ${ }^{89}$ A. A. Komar, ${ }^{95, a}$ Y. Komori, ${ }^{156}$ T. Kondo,${ }^{65}$ K. Köneke, ${ }^{48}$ A. C. König, ${ }^{105}$ T. Kono, ${ }^{65, s}$

R. Konoplich, ${ }^{109, t}$ N. Konstantinidis, ${ }^{77}$ R. Kopeliansky, ${ }^{153}$ S. Koperny ${ }^{38 a}$ L. Köpke, ${ }^{82}$ A. K. Kopp, ${ }^{48}$ K. Korcyl, ${ }^{39}$ K. Kordas, ${ }^{155}$ A. Korn, ${ }^{46}$ A. A. Korol, ${ }^{108}$ I. Korolkov, ${ }^{12}$ E. V. Korolkova, ${ }^{140}$ V. A. Korotkov, ${ }^{129}$ O. Kortner, ${ }^{100}$ S. Kortner, ${ }^{100}$ V. V. Kostyukhin, ${ }^{21}$ S. Kotov, ${ }^{100}$ V. M. Kotov,${ }^{64}$ A. Kotwal, ${ }^{45}$ C. Kourkoumelis, ${ }^{9}$ V. Kouskoura, ${ }^{155}$ A. Koutsman, ${ }^{160 a}$ R. Kowalewski, ${ }^{170}$ T. Z. Kowalski, ${ }^{38 a}$ W. Kozanecki, ${ }^{137}$ A. S. Kozhin, ${ }^{129}$ V. Kral, ${ }^{127}$ V. A. Kramarenko, ${ }^{98}$ G. Kramberger ${ }^{74}$ D. Krasnopevtsev, ${ }^{97}$ M. W. Krasny, ${ }^{79}$ A. Krasznahorkay, ${ }^{30}$ J. K. Kraus, ${ }^{21}$ A. Kravchenko, ${ }^{25}$ S. Kreiss, ${ }^{109}$ J. Kretzschmar, ${ }^{73}$ K. Kreutzfeldt,${ }^{52}$ N. Krieger, ${ }^{54}$ P. Krieger,${ }^{159}$ K. Kroeninger, ${ }^{54}$ H. Kroha, ${ }^{100}$ J. Kroll, ${ }^{121}$ J. Kroseberg, ${ }^{21}$ J. Krstic, ${ }^{13 a}$ U. Kruchonak, ${ }^{64}$ H. Krüger, ${ }^{21}$ T. Kruker,${ }^{17}$ N. Krumnack, ${ }^{63}$ Z. V. Krumshteyn, ${ }^{64}$ A. Kruse,${ }^{174}$ M. C. Kruse, ${ }^{45}$ M. Kruskal, ${ }^{22}$ T. Kubota, ${ }^{87}$ S. Kuday, ${ }^{4 a}$ S. Kuehn, ${ }^{48}$ A. Kugel,${ }^{58 c}$ T. Kuhl, ${ }^{42}$ V. Kukhtin, ${ }^{64}$ Y. Kulchitsky, ${ }^{91}$ S. Kuleshov, ${ }^{32 b}$ M. Kuna, ${ }^{133 a, 133 b}$ J. Kunkle, ${ }^{121}$ A. Kupco, ${ }^{126}$ H. Kurashige, ${ }^{66}$ Y. A. Kurochkin, ${ }^{91}$ R. Kurumida, ${ }^{66}$ V. Kus, ${ }^{126}$ E. S. Kuwertz, ${ }^{148}$ M. Kuze, ${ }^{158}$ J. Kvita, ${ }^{143}$ A. La Rosa ${ }^{49}$ L. La Rotonda ${ }^{37 a, 37 b}$ L. Labarga,${ }^{81}$ S. Lablak, ${ }^{136 a}$ C. Lacasta, ${ }^{168}$ F. Lacava, ${ }^{133 a, 133 b}$ J. Lacey, ${ }^{29}$ H. Lacker, ${ }^{16}$ D. Lacour, ${ }^{79}$ V. R. Lacuesta, ${ }^{168}$ E. Ladygin, ${ }^{64}$ R. Lafaye, ${ }^{5}$ B. Laforge,${ }^{79}$ T. Lagouri, ${ }^{177}$ S. Lai ${ }^{48}$ H. Laier, ${ }^{58 a}$ E. Laisne, ${ }^{55}$ L. Lambourne, ${ }^{77}$ C. L. Lampen, ${ }^{7}$ W. Lampl, ${ }^{7}$ E. Lançon, ${ }^{137}$ U. Landgraf, ${ }^{48}$ M. P. J. Landon, ${ }^{75}$ V. S. Lang, ${ }^{58 a}$ C. Lange, ${ }^{42}$ A. J. Lankford, ${ }^{164}$ F. Lanni, ${ }^{25}$ K. Lantzsch, ${ }^{30}$ A. Lanza, ${ }^{120 a}$ S. Laplace, ${ }^{79}$ C. Lapoire, ${ }^{21}$ J. F. Laporte, ${ }^{137}$ T. Lari, ${ }^{90 a}$ A. Larner, ${ }^{119}$ M. Lassnig, ${ }^{30}$ P. Laurelli, ${ }^{47}$ V. Lavorini, ${ }^{37,37 b}$ W. Lavrijsen, ${ }^{15}$ P. Laycock, ${ }^{73}$ B. T. Le, ${ }^{55}$ O. Le Dortz, ${ }^{79}$ E. Le Guirriec, ${ }^{84}$ E. Le Menedeu, ${ }^{12}$ T. LeCompte, ${ }^{6}$ F. Ledroit-Guillon, ${ }^{55}$ C. A. Lee ${ }^{152}$ H. Lee, ${ }^{106}$ J. S. H. Lee, ${ }^{117}$ S. C. Lee, ${ }^{152}$ L. Lee, ${ }^{177}$ G. Lefebvre, ${ }^{79}$ M. Lefebvre, ${ }^{170}$ F. Legger, ${ }^{99}$ C. Leggett, ${ }^{15}$ A. Lehan, ${ }^{73}$ M. Lehmacher, ${ }^{21}$ G. Lehmann Miotto, ${ }^{30}$ X. Lei, ${ }^{7}$ A. G. Leister, ${ }^{177}$ M. A. L. Leite, ${ }^{24 \mathrm{~d}}$ R. Leitner, ${ }^{128}$ D. Lellouch,${ }^{173}$ B. Lemmer, ${ }^{54}$ K. J. C. Leney, ${ }^{146 c}$ T. Lenz, ${ }^{106}$ G. Lenzen, ${ }^{176}$ B. Lenzi,${ }^{30}$ R. Leone, ${ }^{7}$ K. Leonhardt,${ }^{44}$ S. Leontsinis, ${ }^{10}$ C. Leroy,${ }^{94}$ C. G. Lester, ${ }^{28}$ C. M. Lester, ${ }^{121}$ J. Levêque ${ }^{5}$ D. Levin, ${ }^{88}$ L. J. Levinson, ${ }^{173}$ A. Lewis, ${ }^{119}$ G. H. Lewis, ${ }^{109}$ A. M. Leyko, ${ }^{21}$ M. Leyton, ${ }^{16}$ B. Li,${ }^{33 b, u}$ B. Li ${ }^{84}$ H. Li ${ }^{149}$ H. L. Li ${ }^{31}$ S. Li,${ }^{45}$ X. Li,${ }^{88}$ Z. Liang, ${ }^{119, v}$ H. Liao, ${ }^{34}$ B. Liberti, ${ }^{134 a}$ P. Lichard, ${ }^{30}$ K. Lie, ${ }^{166}$ J. Liebal, ${ }^{21}$ W. Liebig, ${ }^{14}$ C. Limbach, ${ }^{21}$ A. Limosani, ${ }^{87}$ M. Limper ${ }^{62}$ S. C. Lin,${ }^{152, w}$ F. Linde, ${ }^{106}$ B. E. Lindquist,${ }^{149}$ J. T. Linnemann,${ }^{89}$ E. Lipeles, ${ }^{121}$ A. Lipniacka, ${ }^{14}$ M. Lisovyi, ${ }^{42}$ T. M. Liss, ${ }^{166}$ D. Lissauer, ${ }^{25}$ A. Lister, ${ }^{169}$ A. M. Litke, ${ }^{138}$ B. Liu, ${ }^{152}$ D. Liu, ${ }^{152}$ J. B. Liu, ${ }^{33 b}$ 
K. Liu, ${ }^{33 b, x}$ L. Liu, ${ }^{88}$ M. Liu, ${ }^{45}$ M. Liu, ${ }^{33 b}$ Y. Liu, ${ }^{33 b}$ M. Livan, ${ }^{120 a, 120 b}$ S. S. A. Livermore, ${ }^{119}$ A. Lleres, ${ }^{55}$ J. Llorente Merino, ${ }^{81}$ S. L. Lloyd, ${ }^{75}$ F. Lo Sterzo, ${ }^{152}$ E. Lobodzinska, ${ }^{42}$ P. Loch, ${ }^{7}$ W. S. Lockman, ${ }^{138}$ T. Loddenkoetter, ${ }^{21}$ F. K. Loebinger, ${ }^{83}$ A. E. Loevschall-Jensen, ${ }^{36}$ A. Loginov, ${ }^{177}$ C. W. Loh, ${ }^{169}$ T. Lohse, ${ }^{16}$ K. Lohwasser, ${ }^{48}$ M. Lokajicek, ${ }^{126}$ V. P. Lombardo, ${ }^{5}$ J. D. Long, ${ }^{88}$ R. E. Long, ${ }^{71}$ L. Lopes, ${ }^{125 a}$ D. Lopez Mateos, ${ }^{57}$ B. Lopez Paredes, ${ }^{140}$ J. Lorenz, $^{99}$ N. Lorenzo Martinez, ${ }^{116}$ M. Losada, ${ }^{163}$ P. Loscutoff, ${ }^{15}$ M. J. Losty, ${ }^{160 a, a}$ X. Lou, ${ }^{41}$ A. Lounis, ${ }^{116}$ J. Love, ${ }^{6}$ P. A. Love, ${ }^{71}$ A. J. Lowe, ${ }^{144, \mathrm{f}}$ F. Lu, ${ }^{33 \mathrm{a}}$ H. J. Lubatti, ${ }^{139}$ C. Luci, ${ }^{133 a, 133 b}$ A. Lucotte, ${ }^{55}$ D. Ludwig, ${ }^{42}$ I. Ludwig, $^{48}$ F. Luehring, ${ }^{60}$ W. Lukas, ${ }^{61}$ L. Luminari, ${ }^{133 a}$ J. Lundberg, ${ }^{147 a, 147 b}$ O. Lundberg, ${ }^{147 a, 147 b}$ B. Lund-Jensen, ${ }^{148}$ M. Lungwitz, ${ }^{82}$ D. Lynn, ${ }^{25}$ R. Lysak, ${ }^{126}$ E. Lytken, ${ }^{80}$ H. Ma, ${ }^{25}$ L. L. Ma, ${ }^{33 d}$ G. Maccarrone, ${ }^{47}$ A. Macchiolo, ${ }^{100}$ B. Maček, ${ }^{74}$ J. Machado Miguens, ${ }^{125 a, 125 b}$ D. Macina, ${ }^{30}$ R. Mackeprang, ${ }^{36}$ R. Madar, ${ }^{48}$ R. J. Madaras, ${ }^{15}$ H. J. Maddocks, ${ }^{71}$ W. F. Mader, ${ }^{44}$ A. Madsen, ${ }^{167}$ M. Maeno, ${ }^{8}$ T. Maeno, ${ }^{25}$ L. Magnoni, ${ }^{164}$ E. Magradze, ${ }^{54}$ K. Mahboubi, ${ }^{48}$ J. Mahlstedt, ${ }^{106}$ S. Mahmoud, ${ }^{73}$ G. Mahout, ${ }^{18}$ C. Maiani, ${ }^{137}$ C. Maidantchik, ${ }^{24 \mathrm{a}}$ A. Maio, ${ }^{125 a, 125 b, 125 \mathrm{~d}}$ S. Majewski, ${ }^{115}$ Y. Makida, ${ }^{65}$ N. Makovec, ${ }^{116}$ P. Mal, ${ }^{137, y}$ B. Malaescu, ${ }^{79}$ Pa. Malecki, ${ }^{39}$ V. P. Maleev, ${ }^{122}$ F. Malek, ${ }^{55}$ U. Mallik, ${ }^{62}$ D. Malon, ${ }^{6}$ C. Malone, ${ }^{144}$ S. Maltezos, ${ }^{10}$ V. M. Malyshev, ${ }^{108}$ S. Malyukov, ${ }^{30}$ J. Mamuzic, ${ }^{13 b}$ B. Mandelli, ${ }^{30}$ L. Mandelli, ${ }^{90 a}$ I. Mandić, ${ }^{74}$ R. Mandrysch, ${ }^{62}$ J. Maneira, ${ }^{125 a, 125 b}$ A. Manfredini, ${ }^{100}$ L. Manhaes de Andrade Filho, ${ }^{24 b}$ J. A. Manjarres Ramos, ${ }^{137}$ A. Mann, ${ }^{99}$ P. M. Manning, ${ }^{138}$

A. Manousakis-Katsikakis, ${ }^{9}$ B. Mansoulie, ${ }^{137}$ R. Mantifel, ${ }^{86}$ L. Mapelli, ${ }^{30}$ L. March, ${ }^{168}$ J. F. Marchand, ${ }^{29}$ F. Marchese, ${ }^{134 \mathrm{a}, 134 \mathrm{~b}}$ G. Marchiori, ${ }^{79}$ M. Marcisovsky, ${ }^{126}$ C. P. Marino, ${ }^{170}$ C. N. Marques, ${ }^{125 a}$ F. Marroquim, ${ }^{24 a}$ Z. Marshall, ${ }^{15}$ L. F. Marti, ${ }^{17}$ S. Marti-Garcia, ${ }^{168}$ B. Martin, ${ }^{30}$ B. Martin, ${ }^{89}$ J. P. Martin, ${ }^{94}$ T. A. Martin, ${ }^{171}$ V. J. Martin, ${ }^{46}$ B. Martin dit Latour, ${ }^{49}$ H. Martinez, ${ }^{137}$ M. Martinez, ${ }^{12, p}$ S. Martin-Haugh, ${ }^{130}$ A. C. Martyniuk, ${ }^{77}$ M. Marx, ${ }^{139}$ F. Marzano, ${ }^{133 a}$ A. Marzin, ${ }^{112}$ L. Masetti, ${ }^{82}$ T. Mashimo, ${ }^{156}$ R. Mashinistov, ${ }^{95}$ J. Masik, ${ }^{83}$ A. L. Maslennikov, ${ }^{108}$ I. Massa, $^{20 a, 20 b}$ N. Massol, ${ }^{5}$ P. Mastrandrea, ${ }^{149}$ A. Mastroberardino, ${ }^{37 a, 37 b}$ T. Masubuchi, ${ }^{156}$ H. Matsunaga, ${ }^{156}$ T. Matsushita, ${ }^{66}$ P. Mättig, ${ }^{176}$ S. Mättig, ${ }^{42}$ J. Mattmann, ${ }^{82}$ C. Mattravers, ${ }^{119, d}$ J. Maurer, ${ }^{84}$ S. J. Maxfield, ${ }^{73}$ D. A. Maximov, ${ }^{108,9}$ R. Mazini, ${ }^{152}$ L. Mazzaferro, ${ }^{134 a, 134 b}$ M. Mazzanti, ${ }^{90 a}$ G. Mc Goldrick, ${ }^{159}$ S. P. Mc Kee, ${ }^{88}$ A. McCarn, ${ }^{88}$ R. L. McCarthy, ${ }^{149}$ T. G. McCarthy, ${ }^{29}$ N. A. McCubbin, ${ }^{130}$ K. W. McFarlane, ${ }^{56, a}$ J. A. Mcfayden, ${ }^{140}$ G. Mchedlidze, ${ }^{54}$ T. Mclaughlan, ${ }^{18}$ S. J. McMahon, ${ }^{130}$ R. A. McPherson, ${ }^{170, j}$ A. Meade, ${ }^{85}$ J. Mechnich, ${ }^{106}$ M. Mechtel, ${ }^{176}$ M. Medinnis, ${ }^{42}$ S. Meehan, ${ }^{31}$ R. Meera-Lebbai, ${ }^{112}$ S. Mehlhase, ${ }^{36}$ A. Mehta, ${ }^{73}$ K. Meier, ${ }^{58 \mathrm{a}}$ C. Meineck, ${ }^{99}$ B. Meirose ${ }^{80}$ C. Melachrinos, ${ }^{31}$ B. R. Mellado Garcia, ${ }^{146 c}$ F. Meloni, ${ }^{90 a, 90 b}$

L. Mendoza Navas, ${ }^{163}$ A. Mengarelli, ${ }^{20 a, 20 b}$ S. Menke, ${ }^{100}$ E. Meoni, ${ }^{162}$ K. M. Mercurio, ${ }^{57}$ S. Mergelmeyer, ${ }^{21}$ N. Meric, ${ }^{137}$ P. Mermod, ${ }^{49}$ L. Merola, ${ }^{103 a, 103 b}$ C. Meroni, ${ }^{90 a}$ F. S. Merritt, ${ }^{31}$ H. Merritt, ${ }^{110}$ A. Messina, ${ }^{30, z}$ J. Metcalfe, ${ }^{25}$ A. S. Mete, ${ }^{164}$ C. Meyer, ${ }^{82}$ C. Meyer, ${ }^{31}$ J-P. Meyer, ${ }^{137}$ J. Meyer, ${ }^{30}$ J. Meyer, ${ }^{54}$ S. Michal, ${ }^{30}$ R. P. Middleton, ${ }^{130}$ S. Migas, ${ }^{73}$ L. Mijović, ${ }^{137}$ G. Mikenberg, ${ }^{173}$ M. Mikestikova, ${ }^{126}$ M. Mikuž, ${ }^{74}$ D. W. Miller, ${ }^{31}$ C. Mills, ${ }^{57}$ A. Milov, ${ }^{173}$ D. A. Milstead, ${ }^{147 a, 147 b}$ D. Milstein, ${ }^{173}$ A. A. Minaenko, ${ }^{129}$ M. Miñano Moya, ${ }^{168}$ I. A. Minashvili, ${ }^{64}$ A. I. Mincer, ${ }^{109}$ B. Mindur, ${ }^{38 a}$ M. Mineev, ${ }^{64}$ Y. Ming, ${ }^{174}$ L. M. Mir, ${ }^{12}$ G. Mirabelli, ${ }^{133 a}$ T. Mitani, ${ }^{172}$ J. Mitrevski, ${ }^{99}$ V. A. Mitsou, ${ }^{168}$ S. Mitsui, ${ }^{65}$ A. Miucci, ${ }^{49}$ P. S. Miyagawa, ${ }^{140}$ J. U. Mjörnmark, ${ }^{80}$ T. Moa, ${ }^{147 a, 147 b}$ V. Moeller, ${ }^{28}$ S. Mohapatra, ${ }^{149}$ W. Mohr, ${ }^{48}$ S. Molander, ${ }^{147 a, 147 b}$ R. Moles-Valls, ${ }^{168}$ A. Molfetas, ${ }^{30}$ K. Mönig, ${ }^{42}$ C. Monini, ${ }^{55}{ }^{2}$. Monk, ${ }^{36}$ E. Monnier, ${ }^{84}$ J. Montejo Berlingen, ${ }^{12}$ F. Monticelli, ${ }^{70}$ S. Monzani, ${ }^{20 a, 20 b}$ R. W. Moore, ${ }^{3}$ C. Mora Herrera, ${ }^{49}$ A. Moraes, ${ }^{53}$ N. Morange, ${ }^{62} \mathrm{~J}$ Morel, ${ }^{54}$ D. Moreno, ${ }^{82}$ M. Moreno Llácer, ${ }^{168}$ P. Morettini, ${ }^{50 a}$ M. Morgenstern, ${ }^{44}$ M. Morii, ${ }^{57}$ S. Moritz, ${ }^{82}$ A. K. Morley, ${ }^{148}$ G. Mornacchi, ${ }^{30}$ J. D. Morris, ${ }^{75}$ L. Morvaj, ${ }^{102}$ H. G. Moser, ${ }^{100}$ M. Mosidze, ${ }^{51 b}$ J. Moss, ${ }^{110}$ R. Mount, ${ }^{144}$ E. Mountricha, ${ }^{25}$ S. V. Mouraviev, ${ }^{95, a}$ E. J. W. Moyse, ${ }^{85}$ R. D. Mudd, ${ }^{18}$ F. Mueller, ${ }^{58 a}$ J. Mueller $^{124}$ K. Mueller, ${ }^{21}$ T. Mueller, ${ }^{28}$ T. Mueller, ${ }^{82}$ D. Muenstermann, ${ }^{49}$ Y. Munwes, ${ }^{154}$ J. A. Murillo Quijada, ${ }^{18}$ W. J. Murray, ${ }^{171,130}$ I. Mussche, ${ }^{106}$ E. Musto, ${ }^{153}$ A. G. Myagkov, ${ }^{129, a a}$ M. Myska, ${ }^{126}$ O. Nackenhorst, ${ }^{54}$ J. Nadal, ${ }^{54}$ K. Nagai, ${ }^{61}$ R. Nagai, ${ }^{158}$ Y. Nagai, ${ }^{84}$ K. Nagano, ${ }^{65}$ A. Nagarkar, ${ }^{110}$ Y. Nagasaka, ${ }^{59}$ M. Nagel, ${ }^{100}$

A. M. Nairz, ${ }^{30}$ Y. Nakahama, ${ }^{30}$ K. Nakamura, ${ }^{65}$ T. Nakamura, ${ }^{156}$ I. Nakano, ${ }^{111}$ H. Namasivayam, ${ }^{41}$ G. Nanava, ${ }^{21}$ A. Napier, ${ }^{162}$ R. Narayan, ${ }^{58 b}$ M. Nash, ${ }^{77, d}$ T. Nattermann, ${ }^{21}$ T. Naumann, ${ }^{42}$ G. Navarro, ${ }^{163}$ H. A. Neal, ${ }^{88}$ P. Yu. Nechaeva, ${ }^{95}$ T. J. Neep, ${ }^{83}$ A. Negri, ${ }^{120 a, 120 b}$ G. Negri, ${ }^{30}$ M. Negrini, ${ }^{20 \mathrm{a}}$ S. Nektarijevic, ${ }^{49}$ A. Nelson, ${ }^{164}$ T. K. Nelson, ${ }^{144}$ S. Nemecek, ${ }^{126}$ P. Nemethy, ${ }^{109}$ A. A. Nepomuceno, ${ }^{24 a}$ M. Nessi, ${ }^{30, b b}$ M. S. Neubauer, ${ }^{166}$ M. Neumann, ${ }^{176}$ A. Neusiedl, ${ }^{82}$ R. M. Neves, ${ }^{109}$

P. Nevski, ${ }^{25}$ F. M. Newcomer, ${ }^{121}$ P. R. Newman, ${ }^{18}$ D. H. Nguyen, ${ }^{6}$ V. Nguyen Thi Hong, ${ }^{137}$ R. B. Nickerson, ${ }^{119}$ R. Nicolaidou, ${ }^{137}$ B. Nicquevert, ${ }^{30}$ J. Nielsen, ${ }^{138}$ N. Nikiforou, ${ }^{35}$ A. Nikiforov, ${ }^{16}$ V. Nikolaenko, ${ }^{129, \text { aa }}$ I. Nikolic-Audit, ${ }^{79}$ K. Nikolics, ${ }^{49}$ K. Nikolopoulos, ${ }^{18}$ P. Nilsson, ${ }^{8}$ Y. Ninomiya, ${ }^{156}$ A. Nisati, ${ }^{133 a}$ R. Nisius, ${ }^{100}$ T. Nobe, ${ }^{158}$ L. Nodulman, ${ }^{6}$ M. Nomachi, ${ }^{117}$ I. Nomidis, ${ }^{155}$ S. Norberg, ${ }^{112}$ M. Nordberg, ${ }^{30}$ J. Novakova, ${ }^{128}$ M. Nozaki, ${ }^{65}$ L. Nozka, ${ }^{114}$ K. Ntekas, ${ }^{10}$ A.-E. Nuncio-Quiroz, ${ }^{21}$ G. Nunes Hanninger, ${ }^{87}$ T. Nunnemann, ${ }^{99}$ E. Nurse, ${ }^{77}$ F. Nuti, ${ }^{87}$ B. J. O’Brien, ${ }^{46}$ F. O’grady, ${ }^{7}$ D. C. O’Neil, ${ }^{143}$ V. O’Shea, ${ }^{53}$ L. B. Oakes, ${ }^{99}$ F. G. Oakham, ${ }^{29, e}$ H. Oberlack, ${ }^{100}$ J. Ocariz, ${ }^{79}$ A. Ochi, ${ }^{66}$ M. I. Ochoa, ${ }^{77}$ 
S. Oda ${ }^{69}$ S. Odaka, ${ }^{65}$ H. Ogren,${ }^{60}$ A. Oh,${ }^{83}$ S. H. Oh, ${ }^{45}$ C. C. Ohm,${ }^{30}$ T. Ohshima, ${ }^{102}$ W. Okamura, ${ }^{117}$ H. Okawa, ${ }^{25}$ Y. Okumura, ${ }^{31}$ T. Okuyama, ${ }^{156}$ A. Olariu, ${ }^{26 a}$ A. G. Olchevski, ${ }^{64}$ S. A. Olivares Pino, ${ }^{46}$ M. Oliveira, ${ }^{125 a, 125 c, 1}$

D. Oliveira Damazio, ${ }^{25}$ E. Oliver Garcia, ${ }^{168}$ D. Olivito, ${ }^{121}$ A. Olszewski, ${ }^{39}$ J. Olszowska, ${ }^{39}$ A. Onofre, ${ }^{125 a, 125 e}$ P. U. E. Onyisi, ${ }^{31, c c}$ C. J. Oram, ${ }^{160 a}$ M. J. Oreglia, ${ }^{31}$ Y. Oren, ${ }^{154}$ D. Orestano, ${ }^{135 a, 135 b}$ N. Orlando,${ }^{72 a, 72 b}$ C. Oropeza Barrera ${ }^{53}$ R. S. Orr, ${ }^{159}$ B. Osculati, ${ }^{50,50 b}$ R. Ospanov, ${ }^{121}$ G. Otero y Garzon, ${ }^{27}$ H. Otono,${ }^{69}$ M. Ouchrif, ${ }^{136 d}$ E. A. Ouellette, ${ }^{170}$ F. Ould-Saada, ${ }^{118}$ A. Ouraou, ${ }^{137}$ K. P. Oussoren, ${ }^{106}$ Q. Ouyang, ${ }^{33 a}$ A. Ovcharova, ${ }^{15}$ M. Owen, ${ }^{83}$ S. Owen, ${ }^{140}$ V. E. Ozcan, ${ }^{19 a}$ N. Ozturk,${ }^{8}$ K. Pachal,${ }^{119}$ A. Pacheco Pages, ${ }^{12}$ C. Padilla Aranda, ${ }^{12}$ S. Pagan Griso,${ }^{15}$ E. Paganis, ${ }^{140}$ C. Pahl,${ }^{100}$ F. Paige, ${ }^{25}$ P. Pais, ${ }^{85}$ K. Pajchel, ${ }^{118}$ G. Palacino, ${ }^{160 b}$ S. Palestini, ${ }^{30}$ D. Pallin, ${ }^{34}$ A. Palma, ${ }^{125 a, 125 b}$ J. D. Palmer, ${ }^{18}$ Y. B. Pan, ${ }^{174}$ E. Panagiotopoulou, ${ }^{10}$ J. G. Panduro Vazquez,${ }^{76}$ P. Pani,${ }^{106}$ N. Panikashvili, ${ }^{88}$ S. Panitkin, ${ }^{25}$ D. Pantea, ${ }^{26 a}$

Th. D. Papadopoulou, ${ }^{10}$ K. Papageorgiou, ${ }^{155, \mathrm{n}}$ A. Paramonov, ${ }^{6}$ D. Paredes Hernandez, ${ }^{34}$ M. A. Parker, ${ }^{28}$ F. Parodi, ${ }^{50 a, 50 \mathrm{~b}}$ J. A. Parsons, ${ }^{35}$ U. Parzefall, ${ }^{48}$ E. Pasqualucci, ${ }^{133 a}$ S. Passaggio, ${ }^{50 a}$ A. Passeri, ${ }^{135 a}$ F. Pastore, ${ }^{135 a, 135 b, a}$ Fr. Pastore, ${ }^{76}$ G. Pásztor, ${ }^{49, \text { dd }}$ S. Pataraia, ${ }^{176}$ N. D. Patel, ${ }^{151}$ J. R. Pater ${ }^{83}$ S. Patricelli, ${ }^{103 a, 103 b}$ T. Pauly, ${ }^{30}$ J. Pearce,${ }^{170}$ M. Pedersen, ${ }^{118}$ S. Pedraza Lopez, ${ }^{168}$ R. Pedro, ${ }^{125 a, 125 b}$ S. V. Peleganchuk, ${ }^{108}$ D. Pelikan, ${ }^{167}$ H. Peng, ${ }^{33 b}$ B. Penning, ${ }^{31}$ J. Penwell, ${ }^{60}$

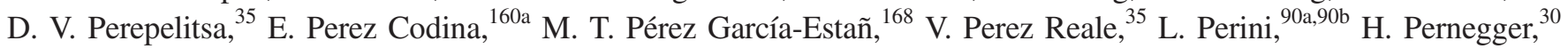
R. Perrino, ${ }^{72 a}$ R. Peschke, ${ }^{42}$ V. D. Peshekhonov,${ }^{64}$ K. Peters, ${ }^{30}$ R. F. Y. Peters,${ }^{54, e e}$ B. A. Petersen, ${ }^{30}$ J. Petersen, ${ }^{30}$ T. C. Petersen ${ }^{36}$ E. Petit, ${ }^{42}$ A. Petridis, ${ }^{147 a, 147 b}$ C. Petridou, ${ }^{155}$ E. Petrolo, ${ }^{133 a}$ F. Petrucci, ${ }^{135 a, 135 b}$ M. Petteni,${ }^{143}$ R. Pezoa, ${ }^{32 b}$ P. W. Phillips,${ }^{130}$ G. Piacquadio, ${ }^{144}$ E. Pianori,${ }^{171}$ A. Picazio,${ }^{49}$ E. Piccaro,${ }^{75}$ M. Piccinini $,{ }^{20 a}, 20 \mathrm{~b}$ S. M. Piec, ${ }^{42}$ R. Piegaia, ${ }^{27}$ D. T. Pignotti, ${ }^{110}$ J. E. Pilcher, ${ }^{31}$ A. D. Pilkington, ${ }^{77}$ J. Pina, ${ }^{125 a, 125 b, 125 d}$ M. Pinamonti, ${ }^{165 a, 165 c, f f}$ A. Pinder, ${ }^{119}$ J. L. Pinfold, ${ }^{3}$ A. Pingel, ${ }^{36}$ B. Pinto, ${ }^{125 a}$ C. Pizio, ${ }^{90 a, 90 b}$ M.-A. Pleier ${ }^{25}$ V. Pleskot, ${ }^{128}$ E. Plotnikova,${ }^{64}$ P. Plucinski, ${ }^{147 a, 147 b}$ S. Poddar, ${ }^{58 a}$ F. Podlyski, ${ }^{34}$ R. Poettgen, ${ }^{82}$ L. Poggioli, ${ }^{116}$ D. Pohl,${ }^{21}$ M. Pohl,${ }^{49}$ G. Polesello, ${ }^{120 a}$ A. Policicchio, ${ }^{37 a, 37 b}$ R. Polifka, ${ }^{159}$ A. Polini, ${ }^{20 a}$ C. S. Pollard, ${ }^{45}$ V. Polychronakos, ${ }^{25}$ D. Pomeroy, ${ }^{23}$ K. Pommès,${ }^{30}$ L. Pontecorvo, ${ }^{133 a}$ B. G. Pope ${ }^{89}$ G. A. Popeneciu, ${ }^{26 b}$ D. S. Popovic, ${ }^{13 a}$ A. Poppleton, ${ }^{30}$ X. Portell Bueso, ${ }^{12}$ G. E. Pospelov, ${ }^{100}$ S. Pospisil, ${ }^{127}$ K. Potamianos, ${ }^{15}$ I. N. Potrap, ${ }^{64}$ C. J. Potter, ${ }^{150}$ C. T. Potter ${ }^{115}$ G. Poulard, ${ }^{30}$ J. Poveda,${ }^{60}$ V. Pozdnyakov, ${ }^{64}$ R. Prabhu ${ }^{77}$ P. Pralavorio, ${ }^{84}$ A. Pranko, ${ }^{15}$ S. Prasad,${ }^{30}$ R. Pravahan,${ }^{8}$ S. Prell,${ }^{63}$ D. Price ${ }^{83}$ J. Price, ${ }^{73}$ L. E. Price, ${ }^{6}$ D. Prieur, ${ }^{124}$ M. Primavera, ${ }^{72 a}$ M. Proissl,${ }^{46}$ K. Prokofiev, ${ }^{109}$ F. Prokoshin, ${ }^{32 b}$ E. Protopapadaki, ${ }^{137}$ S. Protopopescu, ${ }^{25}$ J. Proudfoot, ${ }^{6}$ X. Prudent,${ }^{44}$ M. Przybycien, ${ }^{38 a}$ H. Przysiezniak, ${ }^{5}$ S. Psoroulas, ${ }^{21}$ E. Ptacek, ${ }^{115}$ E. Pueschel,${ }^{85}$ D. Puldon, ${ }^{149}$ M. Purohit, ${ }^{25, g g}$ P. Puzo, ${ }^{116}$ Y. Pylypchenko, ${ }^{62}$ J. Qian, ${ }^{88}$ A. Quadt, ${ }^{54}$ D. R. Quarrie, ${ }^{15}$ W. B. Quayle,${ }^{146 c}$ D. Quilty, ${ }^{53}$ V. Radeka, ${ }^{25}$ V. Radescu, ${ }^{42}$ S. K. Radhakrishnan, ${ }^{149}$ P. Radloff, ${ }^{115}$ F. Ragusa, ${ }^{90 a}{ }^{90 b}$ G. Rahal, ${ }^{179}$ S. Rajagopalan ${ }^{25}$ M. Rammensee,${ }^{48}$ M. Rammes, ${ }^{142}$ A. S. Randle-Conde, ${ }^{40}$ C. Rangel-Smith, ${ }^{79}$ K. Rao, ${ }^{164}$ F. Rauscher, ${ }^{99}$ T. C. Rave, ${ }^{48}$ T. Ravenscroft,${ }^{53}$ M. Raymond ${ }^{30}$ A. L. Read ${ }^{118}$ D. M. Rebuzzi, ${ }^{120 a, 120 b}$ A. Redelbach, ${ }^{175}$ G. Redlinger, ${ }^{25}$ R. Reece,${ }^{138}$ K. Reeves, ${ }^{41}$ L. Rehnisch, ${ }^{16}$ A. Reinsch ${ }^{115}$ H. Reisin, ${ }^{27}$ M. Relich, ${ }^{164}$ C. Rembser ${ }^{30}$ Z. L. Ren, ${ }^{152}$ A. Renaud, ${ }^{116}$ M. Rescigno, ${ }^{133 a}$ S. Resconi, ${ }^{90 a}$ B. Resende, ${ }^{137}$ P. Reznicek, ${ }^{99}$ R. Rezvani, ${ }^{94}$ R. Richter, ${ }^{100}$ M. Ridel,${ }^{79}$ P. Rieck, ${ }^{16}$ M. Rijssenbeek, ${ }^{149}$ A. Rimoldi, ${ }^{120 a, 120 b}$ L. Rinaldi, ${ }^{20 \mathrm{a}}$ E. Ritsch, ${ }^{61}$ I. Riu, ${ }^{12}$ G. Rivoltella, ${ }^{90 a, 90 b}$ F. Rizatdinova, ${ }^{113}$ E. Rizvi, ${ }^{75}$ S. H. Robertson, ${ }^{86, j}$

A. Robichaud-Veronneau, ${ }^{119}$ D. Robinson, ${ }^{28}$ J. E. M. Robinson, ${ }^{83}$ A. Robson, ${ }^{53}$ J. G. Rocha de Lima, ${ }^{107}$ C. Roda, ${ }^{123 a, 123 b}$ D. Roda Dos Santos, ${ }^{126}$ L. Rodrigues, ${ }^{30}$ S. Roe, ${ }^{30}$ O. Røhne, ${ }^{118}$ S. Rolli, ${ }^{162}$ A. Romaniouk, ${ }^{97}$ M. Romano, ${ }^{20 a, 20 b}$ G. Romeo, ${ }^{27}$ E. Romero Adam, ${ }^{168}$ N. Rompotis, ${ }^{139}$ L. Roos,${ }^{79}$ E. Ros, ${ }^{168}$ S. Rosati, ${ }^{133 a}$ K. Rosbach, ${ }^{49}$ A. Rose,${ }^{150}$ M. Rose,${ }^{76}$ P. L. Rosendahl, ${ }^{14}$ O. Rosenthal ${ }^{142}$ V. Rossetti, ${ }^{147 a, 147 b}$ E. Rossi, ${ }^{103 a, 103 b}$ L. P. Rossi, ${ }^{50 a}$ R. Rosten, ${ }^{139}$ M. Rotaru, ${ }^{26 a}$ I. Roth, ${ }^{173}$ J. Rothberg, ${ }^{139}$ D. Rousseau, ${ }^{116}$ C. R. Royon, ${ }^{137}$ A. Rozanov, ${ }^{84}$ Y. Rozen, ${ }^{153}$ X. Ruan, ${ }^{146 c}$ F. Rubbo, ${ }^{12}$ I. Rubinskiy, ${ }^{42}$ V. I. Rud, ${ }^{98}$ C. Rudolph, ${ }^{44}$ M. S. Rudolph ${ }^{159}$ F. Rühr, ${ }^{7}$ A. Ruiz-Martinez, ${ }^{63}$ Z. Rurikova, ${ }^{48}$ N. A. Rusakovich, ${ }^{64}$ A. Ruschke, ${ }^{99}$ J. P. Rutherfoord, ${ }^{7}$ N. Ruthmann, ${ }^{48}$ P. Ruzicka, ${ }^{126}$ Y. F. Ryabov, ${ }^{122}$ M. Rybar, ${ }^{128}$ G. Rybkin, ${ }^{116}$ N. C. Ryder, ${ }^{119}$ A. F. Saavedra, ${ }^{151}$ S. Sacerdoti, ${ }^{27}$ A. Saddique, ${ }^{3}$ I. Sadeh, ${ }^{154}$ H.F-W. Sadrozinski, ${ }^{138}$ R. Sadykov,${ }^{64}$ F. Safai Tehrani, ${ }^{133 a}$ H. Sakamoto, ${ }^{156}$ Y. Sakurai, ${ }^{172}$ G. Salamanna, ${ }^{75}$ A. Salamon, ${ }^{134 a}$ M. Saleem, ${ }^{112}$ D. Salek, ${ }^{106}$ P. H. Sales De Bruin, ${ }^{139}$ D. Salihagic, ${ }^{100}$ A. Salnikov, ${ }^{144}$ J. Salt, ${ }^{168}$ B. M. Salvachua Ferrando, ${ }^{6}$ D. Salvatore, ${ }^{37 a, 37 b}$ F. Salvatore, ${ }^{150}$ A. Salvucci, ${ }^{105}$ A. Salzburger ${ }^{30}$ D. Sampsonidis, ${ }^{155}$ A. Sanchez, ${ }^{103 a, 103 b}$ J. Sánchez, ${ }^{168}$ V. Sanchez Martinez, ${ }^{168}$ H. Sandaker, ${ }^{14}$ H. G. Sander, ${ }^{82}$ M. P. Sanders, ${ }^{99}$ M. Sandhoff, ${ }^{176}$ T. Sandoval, ${ }^{28}$ C. Sandoval, ${ }^{163}$ R. Sandstroem, ${ }^{100}$ D. P. C. Sankey,${ }^{130}$ A. Sansoni, ${ }^{47}$ C. Santoni,${ }^{34}$ R. Santonico, ${ }^{134 a, 134 b}$ H. Santos, ${ }^{125 a}$ I. Santoyo Castillo, ${ }^{150}$ K. Sapp, ${ }^{124}$ A. Sapronov, ${ }^{64}$ J. G. Saraiva, ${ }^{125 a, 125 d}$ E. Sarkisyan-Grinbaum,${ }^{8}$ B. Sarrazin,${ }^{21}$ G. Sartisohn, ${ }^{176}$ O. Sasaki, ${ }^{65}$ Y. Sasaki, ${ }^{156}$ I. Satsounkevitch, ${ }^{91}$ G. Sauvage, ${ }^{5, a}$ E. Sauvan, ${ }^{5}$ J. B. Sauvan, ${ }^{116}$ P. Savard, ${ }^{159, e}$ D. O. Savu, ${ }^{30}$ C. Sawyer, ${ }^{119}$ L. Sawyer, ${ }^{78, o}$ D. H. Saxon, ${ }^{53}$ J. Saxon, ${ }^{121}$ C. Sbarra, ${ }^{20 a}$ A. Sbrizzi, ${ }^{3}$ T. Scanlon, ${ }^{30}$ D. A. Scannicchio, ${ }^{164}$ M. Scarcella, ${ }^{151}$ J. Schaarschmidt, ${ }^{173}$ P. Schacht, ${ }^{100}$ 
D. Schaefer, ${ }^{121}$ A. Schaelicke, ${ }^{46}$ S. Schaepe, ${ }^{21}$ S. Schaetzel, ${ }^{58 b}$ U. Schäfer ${ }^{82}$ A. C. Schaffer,${ }^{116}$ D. Schaile, ${ }^{99}$ R. D. Schamberger, ${ }^{149}$ V. Scharf, ${ }^{58 a}$ V. A. Schegelsky, ${ }^{122}$ D. Scheirich, ${ }^{128}$ M. Schernau, ${ }^{164}$ M. I. Scherzer, ${ }^{35}$ C. Schiavi, ${ }^{50 a, 50 b}$ J. Schieck, ${ }^{99}$ C. Schillo, ${ }^{48}$ M. Schioppa, ${ }^{37,37 b}$ S. Schlenker, ${ }^{30}$ E. Schmidt, ${ }^{48}$ K. Schmieden, ${ }^{30}$ C. Schmitt, ${ }^{82}$ C. Schmitt, ${ }^{99}$ S. Schmitt, ${ }^{58 \mathrm{~b}}$ B. Schneider, ${ }^{17}$ Y. J. Schnellbach, ${ }^{73}$ U. Schnoor,${ }^{44}$ L. Schoeffel, ${ }^{137}$ A. Schoening, ${ }^{58 \mathrm{~b}}$ B. D. Schoenrock, ${ }^{89}$ A. L. S. Schorlemmer, ${ }^{54}$ M. Schott, ${ }^{82}$ D. Schouten, ${ }^{160 a}$ J. Schovancova, ${ }^{25}$ M. Schram, ${ }^{86}$ S. Schramm, ${ }^{159}$ M. Schreyer, ${ }^{175}$ C. Schroeder, ${ }^{82}$ N. Schroer, ${ }^{58 c}$ N. Schuh, ${ }^{82}$ M. J. Schultens, ${ }^{21}$ H.-C. Schultz-Coulon ${ }^{58 a}$ H. Schulz, ${ }^{16}$ M. Schumacher, ${ }^{48}$ B. A. Schumm, ${ }^{138}$ Ph. Schune, ${ }^{137}$ A. Schwartzman, ${ }^{144} \mathrm{Ph}$. Schwegler, ${ }^{100} \mathrm{Ph}$. Schwemling, ${ }^{137}$ R. Schwienhorst,${ }^{89}$ J. Schwindling, ${ }^{137}$ T. Schwindt, ${ }^{21}$ M. Schwoerer, ${ }^{5}$ F. G. Sciacca, ${ }^{17}$ E. Scifo, ${ }^{116}$ G. Sciolla, ${ }^{23}$ W. G. Scott, ${ }^{130}$ F. Scuri, ${ }^{123 a, 123 b}$ F. Scutti, ${ }^{21}$ J. Searcy, ${ }^{88}$ G. Sedov, ${ }^{42}$ E. Sedykh, ${ }^{122}$ S. C. Seidel, ${ }^{104}$ A. Seiden, ${ }^{138}$ F. Seifert, ${ }^{127}$ J. M. Seixas, ${ }^{24 a}$ G. Sekhniaidze, ${ }^{103 a}$ S. J. Sekula, ${ }^{40}$ K. E. Selbach, ${ }^{46}$ D. M. Seliverstov, ${ }^{122}$ G. Sellers, ${ }^{73}$ M. Seman, ${ }^{145 b}$ N. Semprini-Cesari, ${ }^{20,20 b}$ C. Serfon, ${ }^{30}$ L. Serin, ${ }^{116}$ L. Serkin, ${ }^{54}$ T. Serre ${ }^{84}$ R. Seuster, ${ }^{160}$ H. Severini, ${ }^{112}$ F. Sforza, ${ }^{100}$ A. Sfyrla, ${ }^{30}$ E. Shabalina, ${ }^{54}$ M. Shamim, ${ }^{115}$ L. Y. Shan, ${ }^{33 a}$ J. T. Shank, ${ }^{22}$ Q. T. Shao, ${ }^{87}$ M. Shapiro, ${ }^{15}$ P. B. Shatalov, ${ }^{96}$ K. Shaw, ${ }^{165 a, 165 c}$ P. Sherwood, ${ }^{77}$ S. Shimizu, ${ }^{66}$ C. O. Shimmin, ${ }^{164}$ M. Shimojima, ${ }^{101}$ T. Shin, ${ }^{56}$ M. Shiyakova, ${ }^{64}$ A. Shmeleva, ${ }^{95}$ M. J. Shochet, ${ }^{31}$ D. Short, ${ }^{119}$ S. Shrestha, ${ }^{63}$ E. Shulga,${ }^{97}$ M. A. Shupe, ${ }^{7}$ S. Shushkevich, ${ }^{42}$ P. Sicho, ${ }^{126}$ D. Sidorov, ${ }^{113}$ A. Sidoti, ${ }^{133 a}$ F. Siegert, ${ }^{48}$ Dj. Sijacki, ${ }^{13 a}$ O. Silbert, ${ }^{173}$ J. Silva, ${ }^{125 a, 125 d}$ Y. Silver, ${ }^{154}$ D. Silverstein, ${ }^{144}$ S. B. Silverstein, ${ }^{147 a}$ V. Simak, ${ }^{127}$ O. Simard, ${ }^{5}$ Lj. Simic, ${ }^{13 a}$ S. Simion, ${ }^{116}$ E. Simioni, ${ }^{82}$ B. Simmons, ${ }^{77}$ R. Simoniello, ${ }^{90 a}, 90 \mathrm{~b}$ M. Simonyan, ${ }^{36}$ P. Sinervo, ${ }^{159}$ N. B. Sinev, ${ }^{115}$ V. Sipica, ${ }^{142}$ G. Siragusa, ${ }^{175}$ A. Sircar, ${ }^{78}$ A. N. Sisakyan,,${ }^{64, a}$ S. Yu. Sivoklokov, ${ }^{98}$ J. Sjölin, ${ }^{147 a, 147 b}$ T. B. Sjursen, ${ }^{14}$ L. A. Skinnari, ${ }^{15}$ H. P. Skottowe,${ }^{57}$ K. Yu. Skovpen, ${ }^{108}$ P. Skubic, ${ }^{112}$ M. Slater, ${ }^{18}$ T. Slavicek, ${ }^{127}$ K. Sliwa, ${ }^{162}$ V. Smakhtin,${ }^{173}$ B. H. Smart, ${ }^{46}$ L. Smestad, ${ }^{118}$ S. Yu. Smirnov, ${ }^{97}$ Y. Smirnov, ${ }^{97}$ L. N. Smirnova, ${ }^{98, \text { hh }}$ O. Smirnova, ${ }^{80}$ K. M. Smith, ${ }^{53}$ M. Smizanska, ${ }^{71}$ K. Smolek, ${ }^{127}$ A. A. Snesarev, ${ }^{95}$ G. Snidero, ${ }^{75}$ J. Snow, ${ }^{112}$ S. Snyder, ${ }^{25}$ R. Sobie, ${ }^{170, j}$ F. Socher, ${ }^{44}$ J. Sodomka, ${ }^{127}$ A. Soffer, ${ }^{154}$ D. A. Soh, ${ }^{152, v}$ C. A. Solans, ${ }^{30}$ M. Solar, ${ }^{127}$ J. Solc, ${ }^{127}$ E. Yu. Soldatov, ${ }^{97}$ U. Soldevila, ${ }^{168}$ E. Solfaroli Camillocci, ${ }^{133 a, 133 b}$ A. A. Solodkov, ${ }^{129}$ O. V. Solovyanov, ${ }^{129}$

V. Solovyev, ${ }^{122}$ N. Soni, ${ }^{1}$ A. Sood,${ }^{15}$ V. Sopko, ${ }^{127}$ B. Sopko, ${ }^{127}$ M. Sosebee, ${ }^{8}$ R. Soualah, ${ }^{165 a, 165 c}$ P. Soueid,${ }^{94}$ A. M. Soukharev, ${ }^{108}$ D. South, ${ }^{42}$ S. Spagnolo, ${ }^{72 a, 72 b}$ F. Spanò,${ }^{76}$ W. R. Spearman, ${ }^{57}$ R. Spighi, ${ }^{20 a}$ G. Spigo,${ }^{30}$ M. Spousta, ${ }^{128}$ T. Spreitzer, ${ }^{159}$ B. Spurlock, ${ }^{8}$ R. D. St. Denis, ${ }^{53}$ J. Stahlman, ${ }^{121}$ R. Stamen, ${ }^{58 a}$ E. Stanecka, ${ }^{39}$ R. W. Stanek, ${ }^{6}$ C. Stanescu, ${ }^{135 a}$ M. Stanescu-Bellu, ${ }^{42}$ M. M. Stanitzki, ${ }^{42}$ S. Stapnes, ${ }^{118}$ E. A. Starchenko, ${ }^{129}$ J. Stark,${ }^{55}$ P. Staroba, ${ }^{126}$ P. Starovoitov, ${ }^{42}$ R. Staszewski, ${ }^{39}$ P. Stavina, ${ }^{145 a, a}$ G. Steele, ${ }^{53}$ P. Steinbach,${ }^{44}$ P. Steinberg, ${ }^{25}$ I. Stekl, ${ }^{127}$ B. Stelzer, ${ }^{143}$ H. J. Stelzer, ${ }^{89}$ O. Stelzer-Chilton, ${ }^{160 a}$ H. Stenzel, ${ }^{52}$ S. Stern, ${ }^{100}$ G. A. Stewart, ${ }^{30}$ J. A. Stillings,${ }^{21}$ M. C. Stockton, ${ }^{86}$ M. Stoebe, ${ }^{86}$ K. Stoerig, ${ }^{48}$ G. Stoicea, ${ }^{26 a}$ S. Stonjek, ${ }^{100}$ A. R. Stradling, ${ }^{8}$ A. Straessner, ${ }^{44}$ J. Strandberg, ${ }^{148}$ S. Strandberg, ${ }^{147 a, 147 b}$ A. Strandlie, ${ }^{118}$ E. Strauss, ${ }^{144}$ M. Strauss, ${ }^{112}$ P. Strizenec, ${ }^{145 b}$ R. Ströhmer, ${ }^{175}$ D. M. Strom, ${ }^{115}$ R. Stroynowski, ${ }^{40}$ S. A. Stucci, ${ }^{17}$ B. Stugu, ${ }^{14}$ I. Stumer, ${ }^{25, a}$ J. Stupak, ${ }^{149}$ N. A. Styles, ${ }^{42}$ D. Su, ${ }^{144}$ J. Su, ${ }^{124}$ HS. Subramania, ${ }^{3}$ R. Subramaniam, ${ }^{78}$ A. Succurro, ${ }^{12}$ Y. Sugaya, ${ }^{117}$ C. Suhr,${ }^{107}$ M. Suk, ${ }^{127}$ V. V. Sulin, ${ }^{95}$ S. Sultansoy, ${ }^{4 c}$ T. Sumida, ${ }^{67}$ X. Sun,${ }^{55}$ J. E. Sundermann,${ }^{48}$ K. Suruliz, ${ }^{140}$ G. Susinno, ${ }^{37 a, 37 b}$ M. R. Sutton, ${ }^{150}$ Y. Suzuki, ${ }^{65}$ M. Svatos, ${ }^{126}$ S. Swedish, ${ }^{169}$ M. Swiatlowski, ${ }^{144}$ I. Sykora, ${ }^{145 a}$ T. Sykora, ${ }^{128}$ D. Ta, ${ }^{89}$ K. Tackmann, ${ }^{42}$ J. Taenzer, ${ }^{159}$ A. Taffard, ${ }^{164}$ R. Tafirout ${ }^{160 a}$ N. Taiblum, ${ }^{154}$ Y. Takahashi, ${ }^{102}$ H. Takai, ${ }^{25}$ R. Takashima, ${ }^{68}$ H. Takeda, ${ }^{66}$ T. Takeshita, ${ }^{141}$ Y. Takubo, ${ }^{65}$ M. Talby, ${ }^{84}$ A. A. Talyshev, ${ }^{108, g}$ J. Y. C. Tam, ${ }^{175}$ M. C. Tamsett, ${ }^{78, i i}$ K. G. Tan, ${ }^{87}$ J. Tanaka, ${ }^{156}$ R. Tanaka, ${ }^{116}$ S. Tanaka, ${ }^{132}$ S. Tanaka, ${ }^{65}$ A. J. Tanasijczuk, ${ }^{143}$ K. Tani, ${ }^{66}$ N. Tannoury ${ }^{84}$ S. Tapprogge, ${ }^{82}$ S. Tarem,${ }^{153}$ F. Tarrade, ${ }^{29}$ G. F. Tartarelli, ${ }^{90 a}$ P. Tas, ${ }^{128}$ M. Tasevsky, ${ }^{126}$ T. Tashiro, ${ }^{67}$ E. Tassi ${ }^{37 a, 37 \mathrm{~b}}$ A. Tavares Delgado, ${ }^{125 a, 125 \mathrm{~b}}$ Y. Tayalati, ${ }^{136 \mathrm{~d}}$ C. Taylor, ${ }^{77}$ F. E. Taylor,${ }^{93}$ G. N. Taylor, ${ }^{87}$ W. Taylor, ${ }^{160 \mathrm{~b}}$ F. A. Teischinger, ${ }^{30}$ M. Teixeira Dias Castanheira, ${ }^{75}$ P. Teixeira-Dias, ${ }^{76}$ K. K. Temming, ${ }^{48}$ H. Ten Kate, ${ }^{30}$ P. K. Teng, ${ }^{152}$ S. Terada ${ }^{65}$ K. Terashi, ${ }^{156}$ J. Terron,${ }^{81}$ S. Terzo ${ }^{100}$ M. Testa, ${ }^{47}$ R. J. Teuscher, ${ }^{159, j}$ J. Therhaag, ${ }^{21}$ T. Theveneaux-Pelzer, ${ }^{34}$ S. Thoma, ${ }^{48}$ J. P. Thomas, ${ }^{18}$ J. Thomas-Wilsker, ${ }^{76}$ E. N. Thompson, ${ }^{35}$ P. D. Thompson, ${ }^{18}$ P. D. Thompson, ${ }^{159}$ A. S. Thompson, ${ }^{53}$ L. A. Thomsen, ${ }^{36}$ E. Thomson, ${ }^{121}$ M. Thomson, ${ }^{28}$ W. M. Thong, ${ }^{87}$ R. P. Thun, ${ }^{88, a}$ F. Tian, ${ }^{35}$ M. J. Tibbetts, ${ }^{15}$ T. Tic, ${ }^{126}$ V. O. Tikhomirov, ${ }^{95, j j}$ Yu. A. Tikhonov, ${ }^{108, g}$ S. Timoshenko, ${ }^{97}$ E. Tiouchichine,${ }^{84}$ P. Tipton, ${ }^{177}$ S. Tisserant, ${ }^{84}$ T. Todorov, ${ }^{5}$ S. Todorova-Nova, ${ }^{128}$ B. Toggerson,,${ }^{164}$ J. Tojo,${ }^{69}$ S. Tokár, ${ }^{145 a} \mathrm{~K}$. Tokushuku, ${ }^{65} \mathrm{~K}$. Tollefson, ${ }^{89}$ L. Tomlinson, ${ }^{83}$ M. Tomoto, ${ }^{102}$ L. Tompkins,${ }^{31}$ K. Toms, ${ }^{104}$ N. D. Topilin, ${ }^{64}$ E. Torrence, ${ }^{115}$ H. Torres, ${ }^{143}$ E. Torró Pastor, ${ }^{168}$ J. Toth, ${ }^{84, d d}$ F. Touchard ${ }^{84}$ D. R. Tovey, ${ }^{140}$ H. L. Tran, ${ }^{116}$ T. Trefzger, ${ }^{175}$ L. Tremblet, ${ }^{30}$ A. Tricoli, ${ }^{30}$ I. M. Trigger, ${ }^{160 a}$ S. Trincaz-Duvoid, ${ }^{79}$ M. F. Tripiana,${ }^{70}$ N. Triplett,${ }^{25}$ W. Trischuk, ${ }^{159}$ B. Trocmé, ${ }^{55}$ C. Troncon, ${ }^{90 a}$ M. Trottier-McDonald, ${ }^{143}$ M. Trovatelli, ${ }^{135 a, 135 b}$ P. True,${ }^{89}$ M. Trzebinski, ${ }^{39}$ A. Trzupek,${ }^{39}$ C. Tsarouchas, ${ }^{30}$ J.C-L. Tseng, ${ }^{119}$ P. V. Tsiareshka, ${ }^{91}$ D. Tsionou, ${ }^{137}$ G. Tsipolitis, ${ }^{10}$ N. Tsirintanis, ${ }^{9}$ S. Tsiskaridze, ${ }^{12}$ V. Tsiskaridze,${ }^{48}$ E. G. Tskhadadze, ${ }^{51 a}$ I. I. Tsukerman, ${ }^{96}$ 
V. Tsulaia, ${ }^{15}$ J.-W. Tsung, ${ }^{21}$ S. Tsuno, ${ }^{65}$ D. Tsybychev, ${ }^{149}$ A. Tua, ${ }^{140}$ A. Tudorache, ${ }^{26 a}$ V. Tudorache,${ }^{26 a}$ A. N. Tuna, ${ }^{121}$ S. A. Tupputi, ${ }^{20 a, 20 b}$ S. Turchikhin, ${ }^{98, h h}$ D. Turecek, ${ }^{127}$ I. Turk Cakir, ${ }^{4 d}$ R. Turra, ${ }^{90 a, 90 b}$ P. M. Tuts, ${ }^{35}$ A. Tykhonov, ${ }^{74}$ M. Tylmad, ${ }^{147 a, 147 b}$ M. Tyndel, ${ }^{130}$ K. Uchida,${ }^{21}$ I. Ueda,,${ }^{156}$ R. Ueno, ${ }^{29}$ M. Ughetto, ${ }^{84}$ M. Ugland, ${ }^{14}$ M. Uhlenbrock, ${ }^{21}$ F. Ukegawa, ${ }^{161}$ G. Unal, ${ }^{30}$ A. Undrus, ${ }^{25}$ G. Unel,${ }^{164}$ F. C. Ungaro, ${ }^{48}$ Y. Unno, ${ }^{65}$ D. Urbaniec,${ }^{35}$ P. Urquijo, ${ }^{21}$ G. Usai, ${ }^{8}$ A. Usanova, ${ }^{61}$ L. Vacavant, ${ }^{84}$ V. Vacek, ${ }^{127}$ B. Vachon,${ }^{86}$ N. Valencic, ${ }^{106}$ S. Valentinetti, ${ }^{20 a, 20 b}$ A. Valero, ${ }^{168}$ L. Valery, ${ }^{34}$ S. Valkar, ${ }^{128}$ E. Valladolid Gallego, ${ }^{168}$ S. Vallecorsa, ${ }^{49}$ J. A. Valls Ferrer ${ }^{168}$ R. Van Berg, ${ }^{121}$ P. C. Van Der Deijl, ${ }^{106}$ R. van der Geer, ${ }^{106}$ H. van der Graaf, ${ }^{106}$ R. Van Der Leeuw, ${ }^{106}$ D. van der Ster ${ }^{30}$ N. van Eldik, ${ }^{30}$ P. van Gemmeren, ${ }^{6}$ J. Van Nieuwkoop, ${ }^{143}$ I. van Vulpen, ${ }^{106}$ M. C. van Woerden, ${ }^{30}$ M. Vanadia, ${ }^{133 a, 133 b}$ W. Vandelli, ${ }^{30}$ A. Vaniachine, ${ }^{6}$ P. Vankov, ${ }^{42}$ F. Vannucci, ${ }^{79}$ G. Vardanyan, ${ }^{178}$ R. Vari, ${ }^{133 a}$ E. W. Varnes, ${ }^{7}$ T. Varol,,${ }^{85}$ D. Varouchas, ${ }^{15}$ A. Vartapetian, ${ }^{8}$

K. E. Varvell, ${ }^{151}$ V. I. Vassilakopoulos,${ }^{56}$ F. Vazeille, ${ }^{34}$ T. Vazquez Schroeder, ${ }^{54}$ J. Veatch, ${ }^{7}$ F. Veloso, ${ }^{125 a, 125 c}$ S. Veneziano, ${ }^{133 a}$ A. Ventura, ${ }^{72 a, 72 b}$ D. Ventura, ${ }^{85}$ M. Venturi, ${ }^{48}$ N. Venturi, ${ }^{159}$ A. Venturini, ${ }^{23}$ V. Vercesi, ${ }^{120 a}$ M. Verducci, ${ }^{139}$ W. Verkerke, ${ }^{106}$ J. C. Vermeulen, ${ }^{106}$ A. Vest, ${ }^{44}$ M. C. Vetterli, ${ }^{143, e}$ O. Viazlo, ${ }^{80}$ I. Vichou, ${ }^{166}$ T. Vickey, ${ }^{146 c, k k}$ O. E. Vickey Boeriu, ${ }^{146 c}$ G. H. A. Viehhauser,${ }^{119}$ S. Viel, ${ }^{169}$ R. Vigne,${ }^{30}$ M. Villa, ${ }^{20 a, 20 b}$ M. Villaplana Perez, ${ }^{168}$ E. Vilucchi, ${ }^{47}$ M. G. Vincter, ${ }^{29}$ V. B. Vinogradov, ${ }^{64}$ J. Virzi ${ }^{15}$ O. Vitells, ${ }^{173}$ I. Vivarelli, ${ }^{150}$ F. Vives Vaque, ${ }^{3}$ S. Vlachos, ${ }^{10}$ D. Vladoiu, ${ }^{99}$ M. Vlasak, ${ }^{127}$ A. Vogel, ${ }^{21}$ P. Vokac, ${ }^{127}$ G. Volpi, ${ }^{47}$ M. Volpi, ${ }^{87}$ G. Volpini, ${ }^{90 a}$ H. von der Schmitt, ${ }^{100}$ H. von Radziewski, ${ }^{48}$ E. von Toerne, ${ }^{21}$ V. Vorobel, ${ }^{128}$ M. Vos,${ }^{168}$ R. Voss,${ }^{30}$ J. H. Vossebeld, ${ }^{73}$ N. Vranjes, ${ }^{137}$ M. Vranjes Milosavljevic, ${ }^{106}$ V. Vrba, ${ }^{126}$ M. Vreeswijk, ${ }^{106}$ T. Vu Anh, ${ }^{48}$ R. Vuillermet, ${ }^{30}$ I. Vukotic,${ }^{31}$ Z. Vykydal, ${ }^{127}$ W. Wagner, ${ }^{176}$ P. Wagner, ${ }^{21}$ S. Wahrmund, ${ }^{44}$ J. Wakabayashi, ${ }^{102}$ J. Walder, ${ }^{71}$ R. Walker, ${ }^{99}$ W. Walkowiak, ${ }^{142}$ R. Wall, ${ }^{177}$ P. Waller, ${ }^{73}$ B. Walsh, ${ }^{177}$ C. Wang, ${ }^{45}$ H. Wang, ${ }^{15}$ H. Wang, ${ }^{40}$ J. Wang, ${ }^{42}$ J. Wang, ${ }^{33 a}$ K. Wang,${ }^{86}$ R. Wang, ${ }^{104}$ S. M. Wang, ${ }^{152}$ T. Wang, ${ }^{21}$ X. Wang, ${ }^{177}$ A. Warburton, ${ }^{86}$ C. P. Ward ${ }^{28}$ D. R. Wardrope,${ }^{77}$ M. Warsinsky, ${ }^{48}$ A. Washbrook,${ }^{46}$ C. Wasicki, ${ }^{42}$ I. Watanabe, ${ }^{66}$ P. M. Watkins, ${ }^{18}$ A. T. Watson, ${ }^{18}$ I. J. Watson, ${ }^{151}$ M. F. Watson, ${ }^{18}$ G. Watts, ${ }^{139}$ S. Watts, ${ }^{83}$ A. T. Waugh, ${ }^{151}$ B. M. Waugh, ${ }^{77}$ S. Webb,${ }^{83}$ M. S. Weber, ${ }^{17}$ S. W. Weber, ${ }^{175}$ J. S. Webster, ${ }^{31}$ A. R. Weidberg, ${ }^{119}$ P. Weigell, ${ }^{100}$ J. Weingarten, ${ }^{54}$ C. Weiser, ${ }^{48}$ H. Weits, ${ }^{106}$ P. S. Wells,${ }^{30}$ T. Wenaus, ${ }^{25}$ D. Wendland, ${ }^{16}$ Z. Weng, ${ }^{152, v}$ T. Wengler, ${ }^{30}$ S. Wenig, ${ }^{30}$ N. Wermes, ${ }^{21}$ M. Werner, ${ }^{48}$

P. Werner, ${ }^{30}$ M. Wessels, ${ }^{58 \mathrm{a}} \mathrm{J}$. Wetter, ${ }^{162} \mathrm{~K}$. Whalen, ${ }^{29}$ A. White, ${ }^{8} \mathrm{M}$. J. White, ${ }^{1}$ R. White, ${ }^{32 \mathrm{~b}} \mathrm{~S}$. White, ${ }^{123 a, 123 \mathrm{~b}}$ D. Whiteson, ${ }^{164}$ D. Whittington, ${ }^{60}$ D. Wicke, ${ }^{176}$ F. J. Wickens, ${ }^{130}$ W. Wiedenmann, ${ }^{174}$ M. Wielers ${ }^{80, d}$ P. Wienemann, ${ }^{21}$ C. Wiglesworth, ${ }^{36}$ L. A. M. Wiik-Fuchs, ${ }^{21}$ P. A. Wijeratne, ${ }^{77}$ A. Wildauer, ${ }^{100}$ M. A. Wildt,${ }^{42,11}$ H. G. Wilkens, ${ }^{30}$ J. Z. Will, ${ }^{99}$ H. H. Williams, ${ }^{121}$ S. Williams, ${ }^{28}$ W. Willis, ${ }^{35, a}$ S. Willocq, ${ }^{85}$ J. A. Wilson, ${ }^{18}$ A. Wilson, ${ }^{88}$ I. Wingerter-Seez, ${ }^{5}$

S. Winkelmann, ${ }^{48}$ F. Winklmeier, ${ }^{115}$ M. Wittgen, ${ }^{144}$ T. Wittig, ${ }^{43}$ J. Wittkowski, ${ }^{99}$ S. J. Wollstadt, ${ }^{82}$ M. W. Wolter, ${ }^{39}$ H. Wolters, ${ }^{125 a, 125 c}$ W. C. Wong, ${ }^{41}$ B. K. Wosiek, ${ }^{39}$ J. Wotschack, ${ }^{30}$ M. J. Woudstra, ${ }^{83}$ K. W. Wozniak, ${ }^{39}$ K. Wraight, ${ }^{53}$ M. Wright, ${ }^{53}$ S. L. Wu, ${ }^{174}$ X. Wu, ${ }^{49}$ Y. Wu, ${ }^{88}$ E. Wulf,${ }^{35}$ T. R. Wyatt, ${ }^{83}$ B. M. Wynne, ${ }^{46}$ S. Xella ${ }^{36}$ M. Xiao, ${ }^{137}$ D. Xu, ${ }^{33 a}$ L. Xu, ${ }^{33 b, m m}$ B. Yabsley, ${ }^{151}$ S. Yacoob, ${ }^{146 b, n n}$ M. Yamada, ${ }^{65}$ H. Yamaguchi, ${ }^{156}$ Y. Yamaguchi,${ }^{156}$ A. Yamamoto, ${ }^{65}$ K. Yamamoto, ${ }^{63}$ S. Yamamoto, ${ }^{156}$ T. Yamamura, ${ }^{156}$ T. Yamanaka, ${ }^{156}$ K. Yamauchi, ${ }^{102}$ Y. Yamazaki, ${ }^{66}$ Z. Yan, ${ }^{22}$ H. Yang, ${ }^{33 e}$ H. Yang, ${ }^{174}$ U. K. Yang, ${ }^{83}$ Y. Yang, ${ }^{110}$ S. Yanush, ${ }^{92}$ L. Yao, ${ }^{33 a}$ Y. Yasu, ${ }^{65}$ E. Yatsenko, ${ }^{42}$ K. H. Yau Wong, ${ }^{21}$ J. Ye, ${ }^{40}$ S. Ye ${ }^{25}$ A. L. Yen, ${ }^{57}$ E. Yildirim, ${ }^{42}$ M. Yilmaz, ${ }^{4 b}$ R. Yoosoofmiya, ${ }^{124}$ K. Yorita, ${ }^{172}$ R. Yoshida, ${ }^{6}$ K. Yoshihara, ${ }^{156}$ C. Young, ${ }^{144}$ C. J. S. Young, ${ }^{30}$ S. Youssef, ${ }^{22}$ D. R. Yu, ${ }^{15}$ J. Yu ${ }^{8}$ J. M. Yu, ${ }^{88}$ J. Yu, ${ }^{113}$ L. Yuan, ${ }^{66}$ A. Yurkewicz, ${ }^{107}$ B. Zabinski, ${ }^{39}$ R. Zaidan, ${ }^{62}$ A. M. Zaitsev, ${ }^{129, a a}$ A. Zaman, ${ }^{149}$ S. Zambito, ${ }^{23}$ L. Zanello, ${ }^{133 a, 133 b}$ D. Zanzi, ${ }^{100}$ A. Zaytsev, ${ }^{25}$ C. Zeitnitz, ${ }^{176}$ M. Zeman, ${ }^{127}$ A. Zemla ${ }^{38 a}$ K. Zengel,${ }^{23}$ O. Zenin, ${ }^{129}$ T. Ženišs ${ }^{145 a}$ D. Zerwas, ${ }^{116}$ G. Zevi della Porta, ${ }^{57}$ D. Zhang, ${ }^{88}$ H. Zhang, ${ }^{89}$ J. Zhang, ${ }^{6}$ L. Zhang, ${ }^{152}$ X. Zhang, ${ }^{33 \mathrm{~d}}$ Z. Zhang, ${ }^{116}$ Z. Zhao, ${ }^{33 \mathrm{~b}}$ A. Zhemchugov, ${ }^{64}$ J. Zhong, ${ }^{119}$ B. Zhou, ${ }^{88}$ L. Zhou, ${ }^{35}$ N. Zhou, ${ }^{164}$ C. G. Zhu, ${ }^{33 \mathrm{~d}}$ H. Zhu, ${ }^{33 a}$ J. Zhu, ${ }^{88}$ Y. Zhu, ${ }^{33 b}$ X. Zhuang, ${ }^{33 a}$ A. Zibell, ${ }^{99}$ D. Zieminska, ${ }^{60}$ N. I. Zimine, ${ }^{64}$ C. Zimmermann, ${ }^{82}$ R. Zimmermann, ${ }^{21}$ S. Zimmermann, ${ }^{21}$ S. Zimmermann, ${ }^{48}$ Z. Zinonos, ${ }^{54}$ M. Ziolkowski, ${ }^{142}$ R. Zitoun, ${ }^{5}$ G. Zobernig, ${ }^{174}$ A. Zoccoli, ${ }^{20 a, 20 b}$ M. zur Nedden, ${ }^{16}$ G. Zurzolo, ${ }^{103 a, 103 b}$ V. Zutshi ${ }^{107}$ and L. Zwalinski ${ }^{30}$

(ATLAS Collaboration)

${ }^{1}$ School of Chemistry and Physics, University of Adelaide, Adelaide, Australia ${ }_{2}^{2}$ Physics Department, SUNY Albany, Albany, New York, USA

${ }^{3}$ Department of Physics, University of Alberta, Edmonton, Alberta, Canada

${ }^{4 a}$ Department of Physics, Ankara University, Ankara, Turkey

${ }^{4 b}$ Department of Physics, Gazi University, Ankara, Turkey 
${ }^{4 \mathrm{c}}$ Division of Physics, TOBB University of Economics and Technology, Ankara, Turkey

${ }^{4 \mathrm{~d}}$ Turkish Atomic Energy Authority, Ankara, Turkey

${ }^{5}$ LAPP, CNRS/IN2P3 and Université de Savoie, Annecy-le-Vieux, France

${ }^{6}$ High Energy Physics Division, Argonne National Laboratory, Argonne, Illinois, USA

${ }^{7}$ Department of Physics, University of Arizona, Tucson, Arizona, USA

${ }^{8}$ Department of Physics, The University of Texas at Arlington, Arlington, Texas, USA

${ }^{9}$ Physics Department, University of Athens, Athens, Greece

${ }^{10}$ Physics Department, National Technical University of Athens, Zografou, Greece

${ }^{11}$ Institute of Physics, Azerbaijan Academy of Sciences, Baku, Azerbaijan

${ }^{12}$ Institut de Física d'Altes Energies and Departament de Física de la Universitat Autònoma de Barcelona, Barcelona, Spain

${ }^{13 a}$ Institute of Physics, University of Belgrade, Belgrade, Serbia

${ }^{13 \mathrm{~b}}$ Vinca Institute of Nuclear Sciences, University of Belgrade, Belgrade, Serbia

${ }^{14}$ Department for Physics and Technology, University of Bergen, Bergen, Norway

${ }^{15}$ Physics Division, Lawrence Berkeley National Laboratory and University of California, Berkeley, California, USA

${ }^{16}$ Department of Physics, Humboldt University, Berlin, Germany

${ }^{17}$ Albert Einstein Center for Fundamental Physics and Laboratory for High Energy Physics, University of Bern, Bern, Switzerland

${ }^{18}$ School of Physics and Astronomy, University of Birmingham, Birmingham, United Kingdom

${ }^{19 a}$ Department of Physics, Bogazici University, Istanbul, Turkey

${ }^{19 b}$ Department of Physics, Dogus University, Istanbul, Turkey

${ }^{19 \mathrm{c}}$ Department of Physics Engineering, Gaziantep University, Gaziantep, Turkey

${ }^{20 a}$ INFN Sezione di Bologna, Italy

${ }^{20 \mathrm{~b}}$ Dipartimento di Fisica e Astronomia, Università di Bologna, Bologna, Italy

${ }^{21}$ Physikalisches Institut, University of Bonn, Bonn, Germany

${ }^{22}$ Department of Physics, Boston University, Boston, Massachusetts, USA

${ }^{23}$ Department of Physics, Brandeis University, Waltham, Massachusetts, USA

${ }^{24 a}$ Universidade Federal do Rio De Janeiro COPPE/EE/IF, Rio de Janeiro, Brazil

${ }^{24 \mathrm{~b}}$ Federal University of Juiz de Fora (UFJF), Juiz de Fora, Brazil

${ }^{24 \mathrm{c}}$ Federal University of Sao Joao del Rei (UFSJ), Sao Joao del Rei, Brazil

${ }^{24 \mathrm{~d}}$ Instituto de Fisica, Universidade de Sao Paulo, Sao Paulo, Brazil

${ }^{25}$ Physics Department, Brookhaven National Laboratory, Upton, New York, USA

${ }^{26 a}$ National Institute of Physics and Nuclear Engineering, Bucharest, Romania

${ }^{26 \mathrm{~b}}$ National Institute for Research and Development of Isotopic and Molecular Technologies, Physics Department, Cluj Napoca, Romania

${ }^{26 c}$ University Politehnica Bucharest, Bucharest, Romania

${ }^{26 \mathrm{~d}}$ West University in Timisoara, Timisoara, Romania

${ }^{27}$ Departamento de Física, Universidad de Buenos Aires, Buenos Aires, Argentina

${ }^{28}$ Cavendish Laboratory, University of Cambridge, Cambridge, United Kingdom

${ }^{29}$ Department of Physics, Carleton University, Ottawa, Ontario, Canada

${ }^{30}$ CERN, Geneva, Switzerland

${ }^{31}$ Enrico Fermi Institute, University of Chicago, Chicago, Illinois, USA

${ }^{32 a}$ Departamento de Física, Pontificia Universidad Católica de Chile, Santiago, Chile

${ }^{32 \mathrm{~b}}$ Departamento de Física, Universidad Técnica Federico Santa María, Valparaíso, Chile

${ }^{33 a}$ Institute of High Energy Physics, Chinese Academy of Sciences, Beijing, China

${ }^{33 \mathrm{~b}}$ Department of Modern Physics, University of Science and Technology of China, Anhui, China

${ }^{33 \mathrm{c}}$ Department of Physics, Nanjing University, Jiangsu, China

${ }^{33 \mathrm{~d}}$ School of Physics, Shandong University, Shandong, China

${ }^{33 e}$ Physics Department, Shanghai Jiao Tong University, Shanghai, China

${ }^{34}$ Laboratoire de Physique Corpusculaire, Clermont Université and Université Blaise Pascal and CNRS/IN2P3, Clermont-Ferrand, France

${ }^{35}$ Nevis Laboratory, Columbia University, Irvington, New York, USA

${ }^{36}$ Niels Bohr Institute, University of Copenhagen, Kobenhavn, Denmark

${ }^{37}$ INFN Gruppo Collegato di Cosenza, Italy

${ }^{37 \mathrm{~b}}$ Dipartimento di Fisica, Università della Calabria, Rende, Italy

${ }^{38 a}$ AGH University of Science and Technology, Faculty of Physics and Applied Computer Science, Krakow, Poland

${ }^{38 b}$ Marian Smoluchowski Institute of Physics, Jagiellonian University, Krakow, Poland

${ }^{39}$ The Henryk Niewodniczanski Institute of Nuclear Physics, Polish Academy of Sciences, Krakow, Poland

${ }^{40}$ Physics Department, Southern Methodist University, Dallas, Texas, USA

${ }^{41}$ Physics Department, University of Texas at Dallas, Richardson, Texas, USA

${ }^{42}$ DESY, Hamburg and Zeuthen, Germany

${ }^{43}$ Institut für Experimentelle Physik IV, Technische Universität Dortmund, Dortmund, Germany 
${ }^{44}$ Institut für Kern- und Teilchenphysik, Technische Universität Dresden, Dresden, Germany

${ }^{45}$ Department of Physics, Duke University, Durham, North Carolina, USA

${ }^{46}$ SUPA - School of Physics and Astronomy, University of Edinburgh, Edinburgh, United Kingdom

${ }^{47}$ INFN Laboratori Nazionali di Frascati, Frascati, Italy

${ }^{48}$ Fakultät für Mathematik und Physik, Albert-Ludwigs-Universität, Freiburg, Germany

${ }^{49}$ Section de Physique, Université de Genève, Geneva, Switzerland

${ }^{50}$ INFN Sezione di Genova, Italy

${ }^{50 \mathrm{~b}}$ Dipartimento di Fisica, Università di Genova, Genova, Italy

${ }^{51 a}$ E. Andronikashvili Institute of Physics, Ivane Javakhishvili Tbilisi State University, Tbilisi, Georgia

${ }^{51 \mathrm{~b}}$ High Energy Physics Institute, Tbilisi State University, Tbilisi, Georgia

${ }^{52}$ II Physikalisches Institut, Justus-Liebig-Universität Giessen, Giessen, Germany

${ }^{53}$ SUPA-School of Physics and Astronomy, University of Glasgow, Glasgow, United Kingdom

${ }^{54}$ II Physikalisches Institut, Georg-August-Universität, Göttingen, Germany

${ }^{55}$ Laboratoire de Physique Subatomique et de Cosmologie, Université Joseph Fourier and CNRS/IN2P3 and Institut National Polytechnique de Grenoble, Grenoble, France

${ }^{56}$ Department of Physics, Hampton University, Hampton, Virginia, USA

${ }^{57}$ Laboratory for Particle Physics and Cosmology, Harvard University, Cambridge, Massachusetts, USA

${ }^{58 \mathrm{a}}$ Kirchhoff-Institut für Physik, Ruprecht-Karls-Universität Heidelberg, Heidelberg, Germany

${ }^{58 \mathrm{~b}}$ Physikalisches Institut, Ruprecht-Karls-Universität Heidelberg, Heidelberg, Germany

${ }^{58 c}$ ZITI Institut für technische Informatik, Ruprecht-Karls-Universität Heidelberg, Mannheim, Germany

${ }^{59}$ Faculty of Applied Information Science, Hiroshima Institute of Technology, Hiroshima, Japan

${ }^{60}$ Department of Physics, Indiana University, Bloomington, Indiana, USA

${ }^{61}$ Institut für Astro- und Teilchenphysik, Leopold-Franzens-Universität, Innsbruck, Austria

${ }^{62}$ University of Iowa, Iowa City, Iowa, USA

${ }^{63}$ Department of Physics and Astronomy, Iowa State University, Ames, Iowa, USA

${ }^{64}$ Joint Institute for Nuclear Research, JINR Dubna, Dubna, Russia

${ }^{65}$ KEK, High Energy Accelerator Research Organization, Tsukuba, Japan

${ }^{66}$ Graduate School of Science, Kobe University, Kobe, Japan

${ }^{67}$ Faculty of Science, Kyoto University, Kyoto, Japan

${ }^{68}$ Kyoto University of Education, Kyoto, Japan

${ }^{69}$ Department of Physics, Kyushu University, Fukuoka, Japan

${ }^{70}$ Instituto de Física La Plata, Universidad Nacional de La Plata and CONICET, La Plata, Argentina

${ }^{71}$ Physics Department, Lancaster University, Lancaster, United Kingdom

${ }^{72 \mathrm{a}}$ INFN Sezione di Lecce, Italy

${ }^{72 \mathrm{~b}}$ Dipartimento di Matematica e Fisica, Università del Salento, Lecce, Italy

${ }^{73}$ Oliver Lodge Laboratory, University of Liverpool, Liverpool, United Kingdom

${ }^{74}$ Department of Physics, Jožef Stefan Institute and University of Ljubljana, Ljubljana, Slovenia

${ }^{75}$ School of Physics and Astronomy, Queen Mary University of London, London, United Kingdom

${ }^{76}$ Department of Physics, Royal Holloway University of London, Surrey, United Kingdom

${ }^{77}$ Department of Physics and Astronomy, University College London, London, United Kingdom

${ }^{78}$ Louisiana Tech University, Ruston, Louisiana, USA

${ }^{79}$ Laboratoire de Physique Nucléaire et de Hautes Energies, UPMC and Université Paris-Diderot and CNRS/IN2P3, Paris, France

${ }^{80}$ Fysiska institutionen, Lunds universitet, Lund, Sweden

${ }^{81}$ Departamento de Fisica Teorica C-15, Universidad Autonoma de Madrid, Madrid, Spain

${ }^{82}$ Institut für Physik, Universität Mainz, Mainz, Germany

${ }^{83}$ School of Physics and Astronomy, University of Manchester, Manchester, United Kingdom

${ }^{84}$ CPPM, Aix-Marseille Université and CNRS/IN2P3, Marseille, France

${ }^{85}$ Department of Physics, University of Massachusetts, Amherst, Massachusetts, USA

${ }^{86}$ Department of Physics, McGill University, Montreal, Quebec, Canada

${ }^{87}$ School of Physics, University of Melbourne, Victoria, Australia

${ }^{88}$ Department of Physics, The University of Michigan, Ann Arbor, Michigan, USA

${ }^{89}$ Department of Physics and Astronomy, Michigan State University, East Lansing, Michigan, USA

${ }^{90 \mathrm{a}}$ INFN Sezione di Milano, Italy

${ }^{90 \mathrm{~b}}$ Dipartimento di Fisica, Università di Milano, Milano, Italy

${ }^{91}$ B.I. Stepanov Institute of Physics, National Academy of Sciences of Belarus, Minsk, Republic of Belarus

${ }^{92}$ National Scientific and Educational Centre for Particle and High Energy Physics, Minsk, Republic of Belarus

${ }^{93}$ Department of Physics, Massachusetts Institute of Technology, Cambridge, Massachusetts, USA

${ }^{94}$ Group of Particle Physics, University of Montreal, Montreal, Quebec, Canada

${ }^{95}$ P.N. Lebedev Institute of Physics, Academy of Sciences, Moscow, Russia

${ }^{96}$ Institute for Theoretical and Experimental Physics (ITEP), Moscow, Russia 


\author{
${ }^{97}$ Moscow Engineering and Physics Institute (MEPhI), Moscow, Russia \\ ${ }^{98}$ D.V.Skobeltsyn Institute of Nuclear Physics, M.V.Lomonosov Moscow State University, Moscow, Russia \\ ${ }^{99}$ Fakultät für Physik, Ludwig-Maximilians-Universität München, München, Germany \\ ${ }^{100}$ Max-Planck-Institut für Physik (Werner-Heisenberg-Institut), München, Germany \\ ${ }^{101}$ Nagasaki Institute of Applied Science, Nagasaki, Japan \\ ${ }^{102}$ Graduate School of Science and Kobayashi-Maskawa Institute, Nagoya University, Nagoya, Japan \\ ${ }^{103 a}$ INFN Sezione di Napoli, Italy \\ ${ }^{103 \mathrm{~b}}$ Dipartimento di Scienze Fisiche, Università di Napoli, Napoli, Italy \\ ${ }^{104}$ Department of Physics and Astronomy, University of New Mexico, Albuquerque, New Mexico, USA \\ ${ }^{105}$ Institute for Mathematics, Astrophysics and Particle Physics, Radboud University Nijmegen/Nikhef, Nijmegen, Netherlands \\ ${ }^{106}$ Nikhef National Institute for Subatomic Physics and University of Amsterdam, Amsterdam, Netherlands \\ ${ }^{107}$ Department of Physics, Northern Illinois University, DeKalb, Illinois, USA \\ ${ }^{108}$ Budker Institute of Nuclear Physics, SB RAS, Novosibirsk, Russia \\ ${ }^{109}$ Department of Physics, New York University, New York, New York, USA \\ ${ }^{110}$ Ohio State University, Columbus, Ohio, USA \\ ${ }^{111}$ Faculty of Science, Okayama University, Okayama, Japan \\ ${ }^{112}$ Homer L. Dodge Department of Physics and Astronomy, University of Oklahoma, Norman, Oklahoma, USA \\ ${ }^{113}$ Department of Physics, Oklahoma State University, Stillwater, Oklahoma, USA \\ ${ }^{114}$ Palacký University, RCPTM, Olomouc, Czech Republic \\ ${ }^{115}$ Center for High Energy Physics, University of Oregon, Eugene, Oregon, USA \\ ${ }^{116}$ LAL, Université Paris-Sud and CNRS/IN2P3, Orsay, France \\ ${ }^{117}$ Graduate School of Science, Osaka University, Osaka, Japan \\ ${ }^{118}$ Department of Physics, University of Oslo, Oslo, Norway \\ ${ }^{119}$ Department of Physics, Oxford University, Oxford, United Kingdom \\ ${ }^{120 a}$ INFN Sezione di Pavia, Italy \\ ${ }^{120 \mathrm{~b}}$ Dipartimento di Fisica, Università di Pavia, Pavia, Italy \\ ${ }^{121}$ Department of Physics, University of Pennsylvania, Philadelphia, Pennsylvania, USA \\ ${ }^{122}$ Petersburg Nuclear Physics Institute, Gatchina, Russia \\ ${ }^{123 a}$ INFN Sezione di Pisa, Italy \\ ${ }^{123 b}$ Dipartimento di Fisica E. Fermi, Università di Pisa, Pisa, Italy \\ ${ }^{124}$ Department of Physics and Astronomy, University of Pittsburgh, Pittsburgh, Pennsylvania, USA \\ ${ }^{125 a}$ Laboratorio de Instrumentacao e Fisica Experimental de Particulas - LIP, Lisboa, Portugal \\ ${ }^{125 \mathrm{~b}}$ Faculdade de Ciências, Universidade de Lisboa, Lisboa, Portugal \\ ${ }^{125 \mathrm{c}}$ Department of Physics, University of Coimbra, Coimbra, Portugal \\ ${ }^{125 \mathrm{~d}}$ Centro de Física Nuclear da Universidade de Lisboa, Lisboa, Portugal \\ ${ }^{125 \mathrm{e}}$ Departamento de Fisica, Universidade do Minho, Braga, Portugal \\ ${ }^{125 \mathrm{f}}$ Departamento de Fisica Teorica y del Cosmos and CAFPE, Universidad de Granada, Granada, Spain \\ ${ }^{125 \mathrm{~g}}$ Dep Fisica and CEFITEC of Faculdade de Ciencias e Tecnologia, Universidade Nova de Lisboa, Caparica, Portugal \\ ${ }^{126}$ Institute of Physics, Academy of Sciences of the Czech Republic, Praha, Czech Republic \\ ${ }^{127}$ Czech Technical University in Prague, Praha, Czech Republic \\ ${ }^{128}$ Faculty of Mathematics and Physics, Charles University in Prague, Praha, Czech Republic \\ ${ }^{129}$ State Research Center Institute for High Energy Physics, Protvino, Russia \\ ${ }^{130}$ Particle Physics Department, Rutherford Appleton Laboratory, Didcot, United Kingdom \\ ${ }^{131}$ Physics Department, University of Regina, Regina, Saskatchewan, Canada \\ ${ }^{132}$ Ritsumeikan University, Kusatsu, Shiga, Japan \\ ${ }^{133 a}$ INFN Sezione di Roma I, Italy \\ ${ }^{133 \mathrm{~b}}$ Dipartimento di Fisica, Università La Sapienza, Roma, Italy \\ ${ }^{134 a}$ INFN Sezione di Roma Tor Vergata, Italy \\ ${ }^{134 \mathrm{~b}}$ Dipartimento di Fisica, Università di Roma Tor Vergata, Roma, Italy \\ ${ }^{135 a}$ INFN Sezione di Roma Tre, Italy \\ ${ }^{135 b}$ Dipartimento di Matematica e Fisica, Università Roma Tre, Roma, Italy \\ ${ }^{136 a}$ Faculté des Sciences Ain Chock, Réseau Universitaire de Physique des Hautes Energies - Université Hassan II, \\ Casablanca, Morocco \\ ${ }^{136 \mathrm{~b}}$ Centre National de l'Energie des Sciences Techniques Nucleaires, Rabat, Morocco \\ ${ }^{136 c}$ Faculté des Sciences Semlalia, Université Cadi Ayyad, LPHEA-Marrakech, Morocco \\ ${ }^{136 \mathrm{~d}}$ Faculté des Sciences, Université Mohamed Premier and LPTPM, Oujda, Morocco \\ ${ }^{136 \mathrm{e}}$ Faculté des sciences, Université Mohammed V-Agdal, Rabat, Morocco \\ ${ }^{137}$ DSM/IRFU (Institut de Recherches sur les Lois Fondamentales de l'Univers), \\ CEA Saclay (Commissariat à l'Energie Atomique et aux Energies Alternatives), Gif-sur-Yvette, France \\ ${ }^{138}$ Santa Cruz Institute for Particle Physics, University of California Santa Cruz, Santa Cruz, California, USA
}


${ }^{139}$ Department of Physics, University of Washington, Seattle, Washington, USA

${ }^{140}$ Department of Physics and Astronomy, University of Sheffield, Sheffield, United Kingdom

${ }^{141}$ Department of Physics, Shinshu University, Nagano, Japan

${ }^{142}$ Fachbereich Physik, Universität Siegen, Siegen, Germany

${ }^{143}$ Department of Physics, Simon Fraser University, Burnaby, British Columbia, Canada

${ }^{144}$ SLAC National Accelerator Laboratory, Stanford, California, USA

${ }^{145 a}$ Faculty of Mathematics, Physics \& Informatics, Comenius University, Bratislava, Slovak Republic

${ }^{145 \mathrm{~b}}$ Department of Subnuclear Physics, Institute of Experimental Physics of the Slovak Academy of Sciences, Kosice, Slovak Republic

${ }^{146 a}$ Department of Physics, University of Cape Town, Cape Town, South Africa

${ }^{146 \mathrm{~b}}$ Department of Physics, University of Johannesburg, Johannesburg, South Africa

${ }^{146 \mathrm{c}}$ School of Physics, University of the Witwatersrand, Johannesburg, South Africa

${ }^{147 a}$ Department of Physics, Stockholm University, Sweden

${ }^{147 \mathrm{~b}}$ The Oskar Klein Centre, Stockholm, Sweden

${ }^{148}$ Physics Department, Royal Institute of Technology, Stockholm, Sweden

${ }^{149}$ Departments of Physics \& Astronomy and Chemistry, Stony Brook University, Stony Brook, New York, USA

${ }^{150}$ Department of Physics and Astronomy, University of Sussex, Brighton, United Kingdom

${ }^{151}$ School of Physics, University of Sydney, Sydney, Australia

${ }^{152}$ Institute of Physics, Academia Sinica, Taipei, Taiwan

${ }^{153}$ Department of Physics, Technion: Israel Institute of Technology, Haifa, Israel

${ }^{154}$ Raymond and Beverly Sackler School of Physics and Astronomy, Tel Aviv University, Tel Aviv, Israel

${ }^{155}$ Department of Physics, Aristotle University of Thessaloniki, Thessaloniki, Greece

${ }^{156}$ International Center for Elementary Particle Physics and Department of Physics, The University of Tokyo, Tokyo, Japan

${ }^{157}$ Graduate School of Science and Technology, Tokyo Metropolitan University, Tokyo, Japan

${ }^{158}$ Department of Physics, Tokyo Institute of Technology, Tokyo, Japan

${ }^{159}$ Department of Physics, University of Toronto, Toronto, Ontario, Canada

${ }^{160 a}$ TRIUMF, Vancouver, British Columbia, Canada

${ }^{160 \mathrm{~b}}$ Department of Physics and Astronomy, York University, Toronto, Ontario, Canada

${ }^{161}$ Faculty of Pure and Applied Sciences, University of Tsukuba, Tsukuba, Japan

${ }^{162}$ Department of Physics and Astronomy, Tufts University, Medford, Massachusetts, USA

${ }^{163}$ Centro de Investigaciones, Universidad Antonio Narino, Bogota, Colombia

${ }^{164}$ Department of Physics and Astronomy, University of California Irvine, Irvine, California, USA

${ }^{165 a}$ INFN Gruppo Collegato di Udine, Italy

${ }^{165 \mathrm{~b}}$ ICTP, Trieste, Italy

${ }^{165 \mathrm{c}}$ Dipartimento di Chimica, Fisica e Ambiente, Università di Udine, Udine, Italy

${ }^{166}$ Department of Physics, University of Illinois, Urbana, Illinois, USA

${ }^{167}$ Department of Physics and Astronomy, University of Uppsala, Uppsala, Sweden

${ }^{168}$ Instituto de Física Corpuscular (IFIC) and Departamento de Física Atómica, Molecular y Nuclear and Departamento de Ingeniería Electrónica and Instituto de Microelectrónica de Barcelona (IMB-CNM), University of Valencia and CSIC, Valencia, Spain

${ }^{169}$ Department of Physics, University of British Columbia, Vancouver, British Columbia, Canada

${ }^{170}$ Department of Physics and Astronomy, University of Victoria, Victoria, British Columbia, Canada

${ }^{171}$ Department of Physics, University of Warwick, Coventry, United Kingdom

${ }^{172}$ Waseda University, Tokyo, Japan

${ }^{173}$ Department of Particle Physics, The Weizmann Institute of Science, Rehovot, Israel

${ }^{174}$ Department of Physics, University of Wisconsin, Madison, Wisconsin, USA

${ }^{175}$ Fakultät für Physik und Astronomie, Julius-Maximilians-Universität, Würzburg, Germany

${ }^{176}$ Fachbereich C Physik, Bergische Universität Wuppertal, Wuppertal, Germany

${ }^{177}$ Department of Physics, Yale University, New Haven, Connecticut, USA

${ }^{178}$ Yerevan Physics Institute, Yerevan, Armenia

${ }^{179}$ Centre de Calcul de l'Institut National de Physique Nucléaire et de Physique des Particules (IN2P3), Villeurbanne, France

${ }^{\mathrm{a}}$ Deceased.

${ }^{\mathrm{b}}$ Also at Department of Physics, King's College London, London, United Kingdom.

${ }^{c}$ Also at Institute of Physics, Azerbaijan Academy of Sciences, Baku, Azerbaijan.

${ }^{\mathrm{d}}$ Also at Particle Physics Department, Rutherford Appleton Laboratory, Didcot, United Kingdom.

${ }^{\mathrm{e}}$ Also at TRIUMF, Vancouver, British Columbia, Canada.

${ }^{\mathrm{f}}$ Also at Department of Physics, California State University, Fresno, CA, USA.

${ }^{g}$ Also at Novosibirsk State University, Novosibirsk, Russia.

${ }^{\mathrm{h}}$ Also at CPPM, Aix-Marseille Université and CNRS/IN2P3, Marseille, France.

${ }^{\mathrm{i}}$ Also at Università di Napoli Parthenope, Napoli, Italy.

${ }^{\mathrm{j}}$ Also at Institute of Particle Physics (IPP), Canada. 
${ }^{\mathrm{k}}$ Also at Department of Physics, Middle East Technical University, Ankara, Turkey.

${ }^{1}$ Also at Department of Physics, University of Coimbra, Coimbra, Portugal.

${ }^{\mathrm{m}}$ Also at Department of Physics and Astronomy, Michigan State University, East Lansing, MI, USA.

${ }^{\mathrm{n}}$ Also at Department of Financial and Management Engineering, University of the Aegean, Chios, Greece.

${ }^{\circ}$ Also at Louisiana Tech University, Ruston, LA, USA.

${ }^{\mathrm{p}}$ Also at Institucio Catalana de Recerca i Estudis Avancats, ICREA, Barcelona, Spain.

${ }^{\mathrm{q}}$ Also at Department of Physics, University of Cape Town, Cape Town, South Africa.

${ }^{\mathrm{r}}$ Also at CERN, Geneva, Switzerland.

${ }^{\mathrm{s}}$ Also at Ochadai Academic Production, Ochanomizu University, Tokyo, Japan.

${ }^{\mathrm{t}}$ Also at Manhattan College, New York, NY, USA.

${ }^{u}$ Also at Institute of Physics, Academia Sinica, Taipei, Taiwan.

${ }^{\mathrm{v}}$ Also at School of Physics and Engineering, Sun Yat-sen University, Guangzhou, China.

${ }^{w}$ Also at Academia Sinica Grid Computing, Institute of Physics, Academia Sinica, Taipei, Taiwan.

${ }^{\mathrm{x}}$ Also at Laboratoire de Physique Nucléaire et de Hautes Energies, UPMC and Université Paris-Diderot and CNRS/IN2P3, Paris, France.

${ }^{y}$ Also at School of Physical Sciences, National Institute of Science Education and Research, Bhubaneswar, India.

${ }^{\mathrm{z}}$ Also at Dipartimento di Fisica, Università La Sapienza, Roma, Italy.

${ }^{\text {aa }}$ Also at Moscow Institute of Physics and Technology State University, Dolgoprudny, Russia.

${ }^{\mathrm{bb}}$ Also at Section de Physique, Université de Genève, Geneva, Switzerland.

${ }^{\mathrm{cc}}$ Also at Department of Physics, The University of Texas at Austin, Austin, TX, USA.

${ }^{\mathrm{dd}}$ Also at Institute for Particle and Nuclear Physics, Wigner Research Centre for Physics, Budapest, Hungary.

${ }^{e e}$ Also at DESY, Hamburg and Zeuthen, Germany.

${ }^{\mathrm{ff}}$ Also at International School for Advanced Studies (SISSA), Trieste, Italy.

${ }^{g g}$ Also at Department of Physics and Astronomy, University of South Carolina, Columbia, SC, USA.

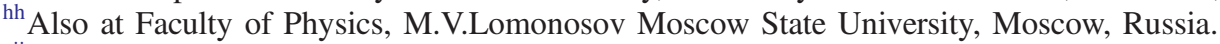

${ }^{\text {ii } A l s o ~ a t ~ P h y s i c s ~ D e p a r t m e n t, ~ B r o o k h a v e n ~ N a t i o n a l ~ L a b o r a t o r y, ~ U p t o n, ~ N Y, ~ U S A . ~}$

${ }^{\mathrm{jj}}$ Also at Moscow Engineering and Physics Institute (MEPhI), Moscow, Russia.

${ }^{\mathrm{kk}}$ Also at Department of Physics, Oxford University, Oxford, United Kingdom.

${ }^{11}$ Also at Institut für Experimentalphysik, Universität Hamburg, Hamburg, Germany.

${ }^{\mathrm{mm}}$ Also at Department of Physics, The University of Michigan, Ann Arbor, MI, USA.

${ }^{n n}$ Also at Discipline of Physics, University of KwaZulu-Natal, Durban, South Africa. 